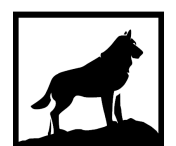

Michigan

Technological

1 8 8 5 University
Michigan Technological University

Digital Commons @ Michigan Tech

\title{
LOCATION, ORBIT AND ENERGY OF A METEOROID IMPACTING THE MOON DURING THE LUNAR ECLIPSE OF JANUARY 21, 2019 \& TESTING THE WEAK EQUIVALENCE PRINCIPLE WITH COSMOLOGICAL GAMMA RAY BURSTS
}

Matipon Tangmatitham

Michigan Technological University, mtangmat@mtu.edu

Copyright 2019 Matipon Tangmatitham

\section{Recommended Citation}

Tangmatitham, Matipon, "LOCATION, ORBIT AND ENERGY OF A METEOROID IMPACTING THE MOON DURING THE LUNAR ECLIPSE OF JANUARY 21, 2019 \& TESTING THE WEAK EQUIVALENCE PRINCIPLE WITH COSMOLOGICAL GAMMA RAY BURSTS", Open Access Dissertation, Michigan Technological University, 2019.

https://doi.org/10.37099/mtu.dc.etdr/834

Follow this and additional works at: https://digitalcommons.mtu.edu/etdr

Part of the Cosmology, Relativity, and Gravity Commons, and the The Sun and the Solar System Commons 


\title{
LOCATION, ORBIT AND ENERGY OF A METEOROID IMPACTING THE MOON DURING THE LUNAR ECLIPSE OF JANUARY 21, 2019 \\ $\&$ TESTING THE WEAK EQUIVALENCE PRINCIPLE WITH COSMOLOGICAL GAMMA RAY BURSTS
}

\author{
By \\ Matipon Tangmatitham

\begin{abstract}
A DISSERTATION
Submitted in partial fulfillment of the requirements for the degree of DOCTOR OF PHILOSOPHY In Physics
\end{abstract}

MICHIGAN TECHNOLOGICAL UNIVERSITY 2019

(C) 2019 Matipon Tangmatitham 
This dissertation has been approved in partial fulfillment of the requirements for the Degree of DOCTOR OF PHILOSOPHY in Physics.

Department of Physics
Dissertation Advisor:
Dr. Robert J. Nemiroff
Committee Member:
Dr. Petra H. Huentemeyer
Committee Member:
Dr. Yang Yang
Committee Member::
Dr. Bijunath R. Patla
Department Chair:
Dr. Ravindra Pandey 


\section{Contents}

List of figures . . . . . . . . . . . . . . . . . vii

List of tables . . . . . . . . . . . . . . . . . . . viii

Preface .............................. . . . .

Acknowledgments ..................... . . . . . . .

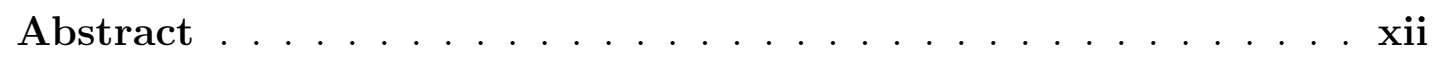

1 Introduction . . . . . . . . . . . . . . . . . 1

2 Location, orbit and energy of a meteoroid impacting the moon during the Lunar Eclipse of January 21, $2019 \ldots \ldots \ldots$

2.1 Introduction . . . . . . . . . . . . . . . . . . . . 6

2.2 Observation of Moon impacts $\ldots \ldots \ldots \ldots$

2.3 Data . . . . . . . . . . . . . . . . . . . . 11

2.4 Location . . . . . . . . . . . . . . . . . . . . 17

2.4 .1 Visual procedure . . . . . . . . . . . . . . . . 17

2.4 .2 Geometrical procedure $\ldots \ldots \ldots \ldots \ldots$

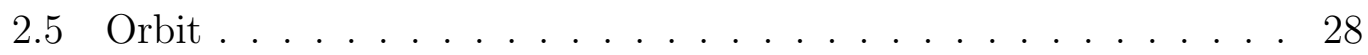

2.6 Energy of the impact $\ldots \ldots \ldots \ldots$

2.6 .1 Photometry . . . . . . . . . . . . . . . . . . . . 32 
2.6 .2 Luminous and kinetic energy . . . . . . . . . . . . . . . 34

2.7 Impactor and crater size . . . . . . . . . . . . . . . . . 35

2.8 Discussion . . . . . . . . . . . . . . . . . . . . . . . . . . 37

3 Testing the Weak Equivalence Principle with Cosmological

Gamma Ray Bursts . . . . . . . . . . . . . . . . . . . . . 42

$3.1 \quad$ Introduction . . . . . . . . . . . . . . . . . . . . . . 43

3.2 Method . . . . . . . . . . . . . . . . . . 46

3.2.1 Statistical Analysis and Confidence Interval . . . . . . . . 47

3.3 Effects of each parameters on limiting WEP violations . . . . . . 48

3.3.1 Comparison with WEP effect on local source . . . . . . . . 49

3.3 .2 Redshift of the Signal. . . . . . . . . . . . . . . . . . . . . 49

3.3 .3 Cutoff radius . . . . . . . . . . . . . . 50

3.3 .4 Cluster Mass Function . . . . . . . . . . . . . . . . . 52

3.4 Results . . . . . . . . . . . . . . . . . . . . 53

4 Conclusion . . . . . . . . . . . . . . . . . . . . . . 58

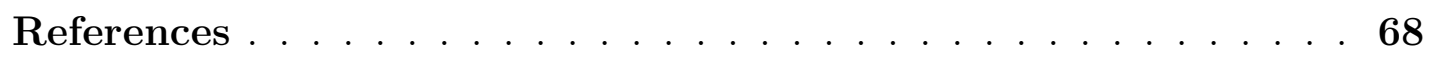

A Copyright documentations . . . . . . . . . . . . . . . . . 69

A.1 Copyright documentation of Chapter 2 . . . . . . . . . . . . . . 69

A.2 Copyright documentation of Chapter 3 . . . . . . . . . . . . . 73 


\section{List of Figures}

$2.1 \quad$ From left to right, frames of the video taken at Ouarzazate, Morocco by Time and Date mobile observatory. The L1-21J flash, which is visible close to the lower dark limb, appeared in four of the six frames. Images reproduced with permission of Time and Date AS. . . . . . . . . . . . . . . . . . . 12

$2.2 \quad$ Picture of the total lunar eclipse at the time of the impact flash taken the observatory LaLoma, Colombia. Picture by Jonathan Ospina, Mauricio Gaviria and Sergio López. . . . . . . . . . . . . 14

2.3 Picture of the total lunar eclipse at the time of the impact flash taken in Santo Domingo, the Dominican Republic. Upper panel: detail of the L1-21J flash. Lower panel: the same picture with the moon removed, highlighting the background stars. 9 stars were identified and used for the parallax and photometry analyses. Picture by Fritz Pichardo. . . . . . . . . . . . . . . . . . . . . . 16

2.4 Craters and lunar features used by the Geometrical procedure in calibrating for the selenographic coordinates (section 2.4.2). Picture by Petr Horálek. . . . . . . . . . . . . . . . . . . . . . . . 18 
2.5 Impact estimated location. Upper row: original picture taken at LaLoma observatory in Colombia (left) and superposition of the original flash image and a LROC ortographic map (right). Bottom row: image of the impact flash superimposed to a high resolution LROC cylindrical map (left) and error regions of the visual and geometrical procedures (right). . . . . . . . . . . . . . . . . . 24

$2.6 \quad$ Overlay of all the apparent lunar position as observed by different observers. The angle differences between each images are used in Parallax calculations (section 2.4.2). All the images are rearranged so that the background stars are alligned. Some reference stars used in photometry are displayed. The apparent positions trace a rough geographical location of the observers, with observations from Europe stacked on the bottom right, the Macaronesian (East Atlantic) islands on the right, with Continenal US and the Caribbean on the top left. . . . . . . . . . . . . . . . . . . . 27

2.7 Marginal probability distributions $(\mathrm{mpd})$ of Impact speed (upper panel) and incident angle (lower panel), as computed using a GRT analysis at the time and location of L1-21J event. . . . . . . . . . 30

2.8 Heat maps of the two-dimensional marginal probability distribution of orbital elements of the parent body (left panel) and radiant locations on the sky (right panel). The dotted line in the right panel represent the boundary between the sky and the solid moon at the impact site. The Sun (and the Earth during the total lunar eclipse) are located in the sky above the impact site close to the $\oplus$ symbol. . . . . . . . . . . . . . . . . . . 31 
2.9 Apparent $G^{\prime}$ magnitude as observed by each image with varying exposure time. The intercept at the flash duration $t=0.3 \mathrm{~s}$ provide the estimation of the true flash magnitude. . . . . . . . . 34

2.10 From top to bottom, posterior probability distributions (ppd) of

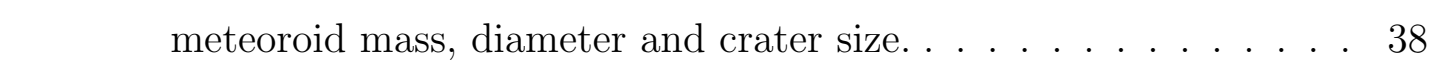

$3.1 \quad$ For a source located at $D_{S}(z)$, each intermediate mass $\left(M_{i}\right)$ near the light path between the observer $(O)$ and the source $(S)$ is placed randomly at comoving cylindrical coordinate $(r, z)=\left(b_{i}, D_{M_{i}}\right) .46$

$3.2 \quad$ Distribution of Shapiro time delay for a source located at $z_{S}=1$ and all clusters within comoving radius of $b_{\max }=10 \mathrm{Mpc}$ are considered. . . . . . . . . . . . . . . . . . . . . . . . . 48

$3.3 \quad$ Relative $\Delta \gamma$ as a function of redshift $z$ of the signal, normalized to $\Delta \gamma(z=1)$. . . . . . . . . . . . . . . . 50

$3.4 \quad$ Relative $\Delta \gamma$ dependence on the maximum impact parameter in comoving coordinate (left), and the same plot expressed in the mass contained within the comoving volume (right), both normalized to $\Delta \gamma\left(b_{\max }=10 \mathrm{Mpc}\right)$ 


\section{List of Tables}

2.1 Properties of the images involved in the analysis of L1-21-J. . . . 13

$2.2 \quad$ Reference stars properties, photometry and astrometry results for the pictures analysed in this work. . . . . . . . . . . . . . . . . . 19

2.3 Continuation of Table 2.2 . . . . . . . . . . . . . . . . . . 20

2.4 Lunar features reference points and their selenographic coordinates, along with the apparent geocentric equatorial coordinate RA, Dec and geocentric distance results from parallax analysis. 21

3.1 A Table of data and WEP-violation limits for rapidly fluctuating and distant GRBs. . . . . . . . . . . . . . . . . . 55 


\section{Preface}

This dissertation consists of two different works on separate areas in astronomy and astrophysics.

The first work on "Location, orbit and energy of a meteoroid impacting the moon during the Lunar Eclipse of January 21, 2019” is a work that is a result of a meteorite impact during the total Lunar Eclipse of January 21, 2019 that has been observed worldwide. In this work, the author has contacted and gathered photographs taken by amateur astronomers across the world who has captured the moment of impact.

The author was originally interested in the citizen science potential in performing an accurate lunar parallax measurements based on amateur astronomer photographs. However, during the analysis the author took note of another work submitted to arXiv regarding the location, orbit, and energy of the meteoroid that caused the impact by Jorge Zuluaga (J.Z.) from University of Antioquia. The author then contacted J.Z. and a collaboration has been formed.

These 6 additional data sets were complimented with J.Z.'s original data. The author used astrometric data among all 7 images were used to calculate the parallax distance to a high precision. The author then develops a geometrical method to further improve the selenographic coordinate of the impact site and was able to achieve a higher constraints compared to the visual method originally performed by J.Z. The author also performed photometric measurements on 6 additional images with different exposures time that allow for a better constraints on the magnitude measurement of the flash over the flash interval.

With these results, the author and J.Z. were able to achieve more accurate results for the impactor's parameters using the gravitational ray tracing technique. Along the way the author and J.Z. collaborated intensely on the revision 
from the original work. Almost every paragraph of the original manuscript has been reworked as a result of this collaboration and we were able to improve our codes that has been made publicly available on Github. With these level of involvement the author was then promoted to the second author in the new version of the manuscript. The complete work has been submitted to the Journal Icarus and in the review process during the time of this dissertation's publication.

The work on "Testing the Weak Equivalence Principle with Cosmological Gamma Ray Bursts" is an original work between M. Tangmatitham and R. Nemiroff. This work attempts to set a new record at constraining the limit to the weak equivalence principle (WEP) violation allowed by current observations. While previous record of WEP violation limit only concerns with gravitational potential from local source of mass, in this work the author try to simulate the contribution from the intermediate clusters of galaxies that are expected to be presented near the light path given current cosmological parameters. Using this novel and original method, the author is able to obtain upper limit that are several order of magnitudes improvement over any other that has been achieved previously in the literature. At the time of writing this dissertation, the work has been submitted to Physical Review Letters and in the process of pending review. 


\section{Acknowledgments}

This has been one heck of a journey. Few graduate students could say they had worked their rear off to the level that I had. It has been nothing short of miraculous how I managed to pull through despite everything. In fact, I still have no idea how I managed it at all.

I could not have done this alone. I would like to thank my family and friends for all the support. If you're reading this: (Yes, YOU) You were there, so you already know how much you have helped me. Time to give yourself a pat in the back. Bravo.

I would also like to give special thanks to my baby boy, whose timely arrival into the world only adds slight complications to this journey. I could never thank my wife enough for taking all the night shifts and let me sleep in peace (but don't tell her I said that). Lastly, I would like to thank my boy again for always enduring his father's little pranks and always be a source of inspirations and funny videos. 


\section{Abstract}

\section{Location, orbit and energy of a meteoroid impacting the moon during the Lunar Eclipse of January 21, 2019}

During the total lunar eclipse of January 21, 2019 at least two meteoroids impacted the moon producing visible flash lights on the near side. One of the impacts occurred on the darkest side of the visible lunar face and was witnessed by many astrophotographers. In this paper we present estimations of the location, impact parameters (velocity and incoming direction), orbit and energy of the meteoroid, as obtained from images and videos collected by amateur astronomers in Colombia, the Dominican Republic, Morocco, USA, Canary Islands, Cape Verde, Czech Republic, Austria, and Germany. Astrometric measurements on the images put the impact location at selenographic lat $=$ $-29.43_{-0.21}^{+0.30}$ and lon. $-67.89_{-0.09}^{+0.07}$ while photometric measurements predict the flash brightness of $G_{f}=6.7 \pm 0.3$. The novel Gravitational Ray Tracing (GRT) technique is used to estimate the orbital properties and radiant of the impactor. We find that that the meteoroid impacted the moon with a speed of $13.8_{-4.3}^{+7.3}$ $\mathrm{km} / \mathrm{s}$ (70\% C.L.) and in a relatively shallow angle, $\theta<38.2$ degrees. According to our photometric estimations, the impact released $6 \times 10^{6} \mathrm{~J}$ of visible light in a short time ( 0.3 seconds). The total impact energy was $\sim 0.5$ tons of TNT which correspond to a body with a mass $\sim 20 \mathrm{~kg}$ and a diameter of $\sim 30 \mathrm{~cm}$. If our assumptions are correct, the crater left by the impact will have $\sim 10$ meters across and it could be detectable by prospecting lunar probes. These results arose from a timely collaboration between professional and amateur astronomers which highlight the importance of citizen science in contemporary astronomy. 


\section{Testing the Weak Equivalence Principle with Cosmological Gamma Ray Bursts}

Gamma Ray Bursts (GRBs) with rapid variations at cosmological distances are used to place new limits on violations of the gravitational weak equivalence principle (WEP). These limits track intrinsic timing deviations between GRB photons of different energies as they cross the universe, in particular in the $\mathrm{KeV}$ to $\mathrm{GeV}$ energy range. Previous limits in this energy range have involved only the gravitational potential of local sources and utilized temporal variability on the order of 0.1 seconds. Here WEP violation limits are derived from sources with greater distance, faster variability, and larger intervening mass. Specifically, GRB sources with redshifts as high as 6.5 are considered, with variability as fast 0.2 milliseconds, and passing the gravitational potentials of inferred clusters of galaxies distributed randomly around the line of sight. WEP violation limits are derived from data from GRB 910711, GRB 920229, GRB 021206, GRB 051221, GRB 090429, and GRB 090510. The strongest constraint in the very early universe comes from GRB 090429 which limits $\gamma(500 \mathrm{keV})-\gamma(250 \mathrm{keV})<$ $1.2 \times 10^{-13}$. The strongest overall constraint comes from GRB 090510 which yields a WEP violation limit of $\gamma(30 \mathrm{GeV})-\gamma(1 \mathrm{GeV})<6.6 \times 10^{-16}$. This strongest constraint is not only a new record for WEP violation limit for gammaray photons and in the early universe, but the strongest upper bound for $\Delta \gamma$ that has ever been recorded between any two energy bands. 


\section{Chapter 1}

\section{Introduction}

This dissertation consisted of 2 separate works from two different disciplines in astrophysics. Chapter 2 is a work on finding the location of the meteoroid impact on the moon during Lunar Eclipse of January 21, 2019. Chapter 3 is a work on testing the weak equivalence principle with cosmological gamma ray bursts.

\section{Location, orbit and energy of a meteoroid im- pacting the moon during the Lunar Eclipse of January 21, 2019}

During the total lunar eclipse of January 21, 2019, a meteoroid impact on the eclipsing side of the moon was observed by millions and recorded to many video camera livestreaming the event. In the subsequent days many images of the impact event were submitted by the amateur astronomers to Astronomy Picture of the Day (APOD) 1]. The author took note from the many different locations the event was observed from and saw a potential for citizen science project.

\footnotetext{
${ }^{1}$ https: //apod.nasa.gov
} 
With personal background in amateur astronomy, astrophotography, citizen science, and doing lunar parallax experiment as part of public outreach, the author noticed a huge potential that this rare event could offer. Normally, lunar parallax can be difficult to obtain to a high precisions due to many difficulties involved: The high dynamic range between full moon and background stars, the difficulties of displacing different observers across vast geographical distances, and the meticulous synchronization required to ensure the images are taken simultaneously.

With the total lunar eclipse, these difficulties were already addressed. The Earth was blocking most of the sunlight, dimming the full moon. There were already a large number of amateur astronomers across vast geographical distances observing at the same time. Moreover, the presence of the flash ensured that all images are synchronized and overlapped to the same fractions of a second.

During the time of the project, a paper by J. Zuluaga et al. on the impact was submitted to arXiv. The author noticed the potential for our combined data and contacted J. Zuluaga. The collaboration between the author and JZ was then formed and as a result we were able to achieve much more comprehensive work and improved upon the previous results.

On top of the Lunar parallax measurements, this collaboration also attempts to learn more about the impact event. Although lunar impacts are relatively common and has been documented before, the impact of January 21, 2019 was the first one detected simultaneously by thousands of observers during a total lunar eclipse. Given the level of involvements and interests shared by general public and amateur astronomers, this event has become a truly historical event and is truly one of its kind, at least for the time being.

In this work the author and the collaborator attempt to perform a scientific analysis of the event using observations gathered by amateur and professional 
astronomers across the globe. From photometry we can learn more about the energy involved in the impact that allows us to explore the mass and size of the impactor together with probable diameter of the newly formed crater. From astrometry of the flash we can narrow down the area where the crater could've been discovered by lunar-orbiting satellite such as the Lunar Reconaissance Orbiter (LRO). Based on the location on the lunar surface we also attempt to estimate the orbit of the meteoroid using a novel Gravitational Ray Tracing (GRT) technique.

\section{Testing the Weak Equivalence Principle with Cos- mological Gamma Ray Bursts}

Einstein's theory of General Relativity has been one of the most impactful theories of physics in the modern era. It has survived many experiments and observations. Regardless, it is likely not the complete picture of how our Universe functions and finding its drawback and the limit to which it fails would offer us one big step closer to the complete understanding of the Universe.

If Einstein's theory of General Relativity has been proven to be incorrect, then the limitations to which it begins to fail will offer us a glimpse to a better and more complete theory to the Universe. This would be analogous to how the limitations in Newton's Law of Universal Gravitation lead Einstein to discover the theory of General Relativity. On the other hand, a non-detection of any violations at the extreme level would strongly suggest that gravity is fundamentally different from the other forces that could lead to an even more radical rethinking[2].

The Weak Equivalence Principle (WEP) is one of the tests for the theory of General Relativity. WEP states that any two free-falling test particles under the 
same gravitational field must follow the same trajectory. Cosmological test for WEP involves observing photons particle of different energies traveling across vast cosmological distances and gravitational field to the Solar System. If two photons that were released simultaneously reached the observer at different time, then WEP would be violated. This experiment is thus equivalent of the Galileo's supposed ball drop experiment atop the tower of Pisa. By observing the smallest $\Delta t$ from sources such as the Gamma Ray Bursts (GRB), we are able to put the upper limit to the amount of WEP violations parameterized by $\Delta \gamma$.

However, all previous limits to $\Delta \gamma$ from cosmological sources only assume the local gravitational field of objects like the Milky Way Galaxy or the Local Cluster of Galaxies. All the intermediate mass along the light path is ignored due to the lack of complete information of the exact locations of clusters in the Universe.

In this work, the author is inspired to explore the scenario if we were to include intermediate mass by assuming homogeneity in the comoving volume along the light path up to $\rho_{\text {crit }} \Omega_{C}$. By simulating clusters of galaxies near the light path, the author was able to prove that the gravitational field and subsequently the Shapiro time delay contribution from these intermediate mass should exceed those of local sources by multiple order of magnitudes. By using this novel technique the author is able to achieve a record breaking upper bound for $\Delta \gamma$ based on current observations. This new record has put a much tighter constraints and offer a better look to how accurate the Einstein's theory of General Relativity can be. 


\title{
Chapter 2
}

\section{Location, orbit and energy of a}

\section{meteoroid impacting the moon}

\section{during the Lunar Eclipse of January 21, 2019!}

\begin{abstract}
During the total lunar eclipse of January 21, 2019 at least two meteoroids impacted the moon producing visible flashes of light on the near side. One of the impacts occurred on the darkest side of the visible lunar face and was witnessed by many casual observers. In this paper, estimations of the location, impact parameters (velocity and incoming direction), orbit and energy of the meteoroid are presented, as obtained from images and videos collected by am-

${ }^{1}$ Work on this chapter has been submitted to the Journal Icarus. Citation: J. Zuluaga, M. Tangmatitham, P. Cuartas-Restrepo, J. Ospina, F. Pichardo, S. López, K. Peña, J. GaviriaPosada (2019). The role and contribution of the author in this work is explained in details in the Preface.
\end{abstract}


ateur astronomers in Colombia, the Dominican Republic, Morocco, USA, Canary Islands, Cape Verde, Czech Republic, Austria, and Germany. Astrometric measurements on the images put the impact location at selenographic lat $=$ $-29.43_{-0.21}^{+0.30}$ and lon. $-67.89_{-0.09}^{+0.07}$ while photometric measurements predict the flash brightness of $G_{f}=6.7 \pm 0.3$. The novel Gravitational Ray Tracing (GRT) technique is used to estimate the orbital properties and radiant of the impactor. We find that that the meteoroid impacted the moon with a speed of $13.8_{-4.3}^{+7.3}$ $\mathrm{km} / \mathrm{s}(70 \%$ C.L.) and in a relatively shallow angle, $\theta<38.2$ degrees. According to our photometric estimations, the impact released $6 \times 10^{6} \mathrm{~J}$ of visible light in a short time ( 0.3 seconds). The total impact energy was $\sim 0.2-1.3$ tons of TNT which correspond to a body with a mass $\sim 7-79 \mathrm{~kg}$ and a diameter of $\sim 18-41 \mathrm{~cm}$. If our assumptions are correct, the crater left by the impact will have $\sim 5-12$ meters across and it could be detectable by prospecting lunar probes. These results arose from a timely collaboration between professional and amateur astronomers which highlight the importance of citizen science in contemporary astronomy.

\section{$2.1 \quad$ Introduction}

In January 21, 2019 the only total lunar eclipse of 2019 took place. Thousands, if not millions of observers, follow the event from all Americas, north Africa and most Europe. As usual, several amateur and professional observatories around the world streamed the whole eclipse over the internet.

Few minutes after the beginning of the totality, several sources on the internet claimed the observation of a short flash of light on the east side of the eclipsed moon. A few hours after, the flash was fully confirmed by the Moon Impacts Detection and Analysis System, MIDAS 3$]$ in Spain. According to MIDAS, one 
meteoroid (hereafter L1-21J) impacted the darker side of the eclipsed moon at 04:41:38 UTC. In the days after the eclipse, the Royal Observatory $2^{2}$ reported a second flash just two minutes after L1-21J occurring on the western and much brighter limb of the eclipsed moon. To the date of writing, however, this second flash has not been confirmed by other observers.

Right after the confirmation by MIDAS of the first impact, several observers around the world reported the independent detection of the flash in their own footage. In this paper, we describe and analyze images provided by amateur and professional astronomers in Colombia, the Dominican Republic, USA, Canary Islands, Cape Verde, Czech Republic, Austria, and Germany (see section 2.3).

Although lunar impacts are relatively common, as it is confirmed by the observation of these almost consecutive events, the impacts of January 21, 2019 are the first ones detected simultaneously by thousands of observers during a total lunar eclipse. This latter condition offers unique opportunities to study this phenomena in exquisite detail.

Here, we present a scientific analysis of the L1-21J event using observations gathered, independently, by amateur and professional astronomers, in the Americas, Africa and Europe. First, We briefly review what we know about impacts of small meteoroids on the moon (Section 2.2). Then, we describe the instruments and data we gather and analyze for this work (Section 2.2). One of the most interesting and unique characteristic of our approach, is the numerical "reconstruction" of the meteoroid trajectory, which is required to estimate its speed and incident angle. For this purpose we use the novel Gravitational Ray Tracing (GRT) techinque (Section 2.5). Photometric analysis of our footage provide us estimations of the total energy involved in the impact (Section 2.6); from there we can estimate the posterior probability distribution (ppd) of the mass and size

\footnotetext{
${ }^{2}$ https://www.rmg.co.uk
} 
of the impactor (Section 2.7). The precise location of the impact and the crater diameter left by the event is also estimated.

\subsection{Observation of Moon impacts}

Impacts on the Earth-Moon system are relatively common [4, 5, 6, 17, 8, 9]. It has been estimated[10] that $\sim 10^{4}$ small, low-mass meteoroids $(\sim 0.1 \mathrm{~m})$ enter into the Earth's atmosphere per year ( 1 impact per hour). The Earth/Moon ratio of meteoroid fluxes is estimated to be 1.38 [8]. Therefore, the rate of impacts on the Moon is similar. However, since our satellite lacks dense atmosphere, the effects of those impacts on its surface are more dramatic and easier to detect.

With the exception of the event described in the chronicles of Gervase of Canterbury in 1178 [11] (whose nature is still debated), the visual observation of impacts on the Moon is not very common. According to [4], those impacts could be observed under three favorable conditions: 1) in the days close to the New moon when the dark side is illuminated only by the Earthshine, 2) far from the dark limb, close to first or last quarter and 3) the most favorable, but far less frequent condition, during a total lunar eclipse. In fact, the first impact recorded during a total lunar eclipse was the one reported by [4] on January 21, 2000 (exactly one metonic cycle ago).

In recent years, with improved optical and electronic astronomical equipment and prospecting lunar satellites, the detection of "fresh" impacts on the moon can be detected using two methods: 1) a local method, involving the observation of the lunar surface from prospecting satellites; and 2) a remote method, which relies on the observation of the short visible flashes during the impacts.

The NASA Lunar Reconnaissance Orbiter (LRO) has succesfully tested the 
first method ${ }^{3}$. During a 6 years mission [12] the LRO has taken high-resolution images (down to 1 meter per pixel) of $70 \%$ of the Moon surface, with almost $3 \%$ of the surface observed at least two times. During that time, the spacecraft has detected signatures of hundreds of fresh impacts. Particularly notorious are two impacts that were first observed from the Earth and afterwards their associated crater was discovered by LRO. The first one was an extremely bright impact happening on march 17, 2013 [13]; the second one happened on september 11, 2013 and it was also identified by the MIDAS system [14]. LRO fresh impact signatures have been used for calibrating the Moon cratering flux and to test theoretical estimations of meteoroid fluxes on the Earth-Moon system [12].

In the last two decades, several observing systems were designed and built to monitor the Moon, looking for flash events. These systems are especially active during the peak of large meteor showers such as the geminids, leonids and perseids [15, 16, 17, 18, 19, 20, 21]. One of the best-known monitor system is MIDAS [3]. MIDAS has been operational for almost two decades; it has detected a significant number of flashes on the Moon, and its data products have been used to study the population properties of major meteor showers [14, 18, 19, 20]. MIDAS was the first of such monitor system confirming the L1-21J event. More recently, the NEO Lunar Impacts and Optical TrAnsients, NELIOTA saw the first light [22]. To date at least 55 flashes have been registered by the NELIOTA team, most of them produced by sporadic impactors and some by geminids.

In contrast to the abundant information available about Earth's impacts (most of them are detected visually or acoustically from the ground and from the space), a limited amount of information about moon impacts can be obtained only from the detection of lunar flashes.

The kinetic energy of the impactor $K$, can be estimated from the luminous

\footnotetext{
$\sqrt[3]{\text { http://target.lroc.asu.edu/output/lroc/lroc_page.html }}$
} 
energy of the flash $E_{r}$, assuming a simple relationship:

$$
E_{r}=\eta K
$$

Here, $\eta$ is the so-called luminous efficiency, a critical but very uncertain quantity. The observation of large fireballs on the Earth's atmosphere has allowed us to constraint the luminous efficiency to $\eta \lesssim 10 \%$ [10]. In the case of impacts on the Moon, the estimation of the distribution of particles diameters in major meteor showers (which depends on their kinetic energy and hence on $\eta$ ), has allowed us to constrain the value of this quantity in the interval $\eta \sim 0.0018-0.0034$, or equivalently $\log \eta=-2.61 \pm 0.14$ [14].

Kinetic energy alone does not allow the precise estimation of other physical properties of the impactor. Speed, mass and composition are, for instance, almost impossible to be measured just from observation of the flashes. However, educated guesses of the speed and incident angle (as obtained from theoretical independent models), provide useful estimations of the meteoroid properties [8]. The success of these estimations was subject to a test after the bright flash on september 11, 2013. Madiedo[14] has estimated that, assuming a luminous efficiency $\eta=0.002$ and an impact speed of $v_{\mathrm{imp}}=17 \mathrm{~km} / \mathrm{s}$, the crater left by the impact will have a diameter of $34 \mathrm{~m}$. The discovery by the LRO of a $46 \mathrm{~m}$ crater in the place of the 2013 intense flash, give some confidence to the theoretical models. However, one or two detections are statistically insufficient to demonstrate, without any doubt, the validity of the models. The detection and analysis of more events will be required to construct improved models. 


\subsection{Data}

Our analysis of the L1-21J are based on several independent observations performed by amateur and professional astronomers around the world that were observing the eclipse for other purposes.

The initial footage that motivated this research was a video taken by the mobile observatory of the timeanddate astronomy portat At the time of the eclipse, the observatory was located in Ouarzazate, Morocco. These observation supported the on-line transmission of the phenomenon in the Time and Date website. From the video, we extracted 6 frames around the time of the flash and analyzed them separately to obtain a light profile of the event and to estimate the flash duration (see Section 2.6.1). In Figure 2.1, we show the selected frames.

Once the time of the impact was precisely determined, many casual observers around the world, looked-up among their images to see if the event was accidentally recorded. Two of us (J.Z. and K.P.) received images and data from amateur astronomers in Colombia and the Dominican Republic. Independently, one of us (M.T.), received additional images via submission to the Astronomy Picture of the Day (APOD) ${ }^{5}[1$ and notice the potential for collective citizen science project, particularly in parallax information from simultaneous observation of the same events. The photographers were then contacted and permission to use their images had been requested and subsequently granted. After reviewing the images, we selected 8 pictures meeting basic criteria of quality and metadata availability required for their proper reduction.

In Table 2.1 we enumerate the properties of all the location from which we obtain pictures.

The picture with the best resolution (that was used for locating the impact

\footnotetext{
${ }^{4}$ https: //www.timeanddate.com/

5 http://apod.nasa.gov
} 


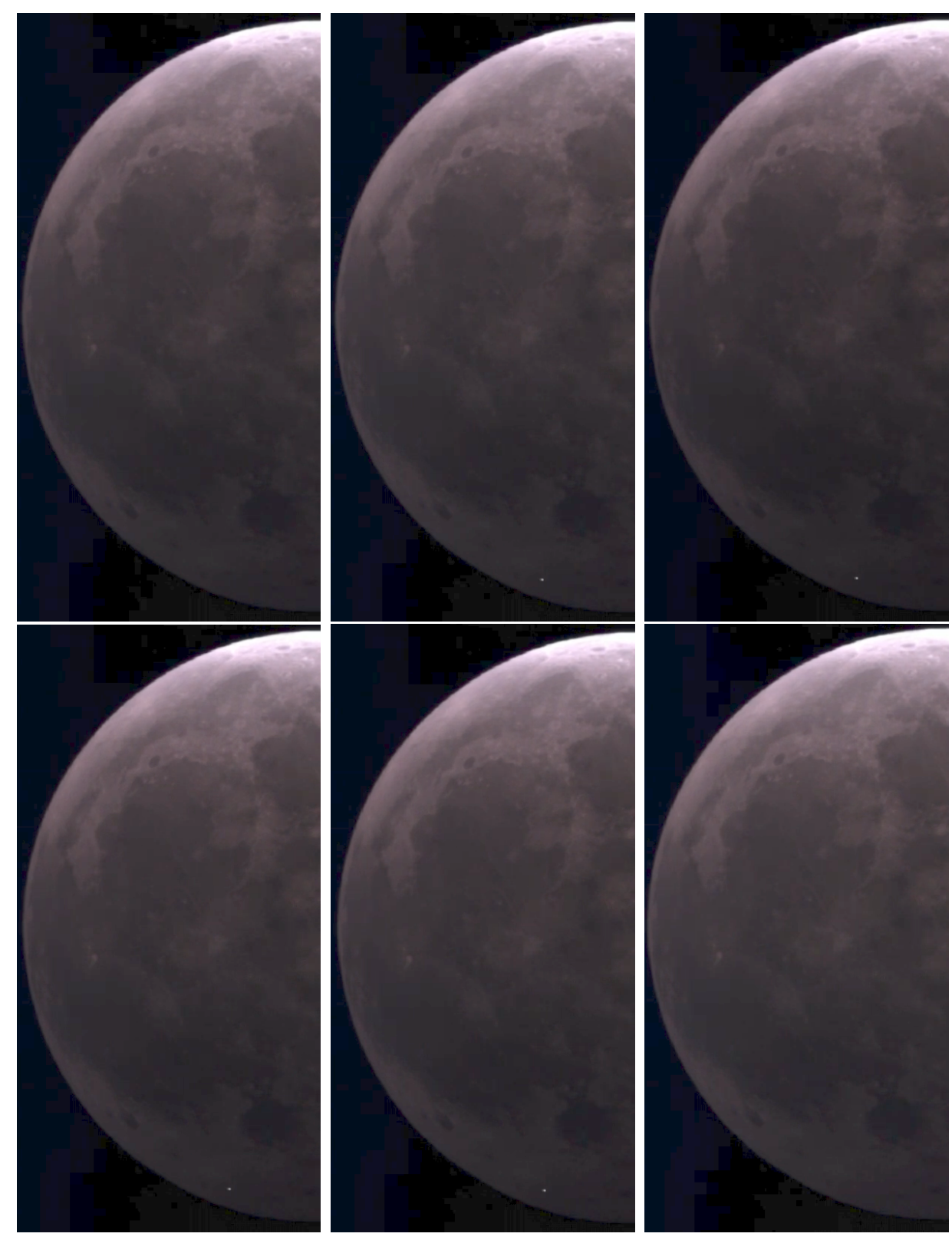

Figure 2.1: From left to right, frames of the video taken at Ouarzazate, Morocco by Time and Date mobile observatory. The L1-21J flash, which is visible close to the lower dark limb, appeared in four of the six frames. Images reproduced with permission of Time and Date AS. 


\begin{tabular}{|c|c|c|c|c|c|c|c|}
\hline Location & $\begin{array}{l}\text { Latitude } \\
\text { (deg) }\end{array}$ & $\begin{array}{l}\text { Longitude } \\
\text { (deg) }\end{array}$ & $\begin{array}{c}\text { Elevation } \\
(\mathrm{m})\end{array}$ & $\begin{array}{l}\text { Exposure time } \\
\text { (seconds) }\end{array}$ & $\begin{array}{c}\text { Flash magnitude }^{\dagger} \\
\text { (G magnitude) }\end{array}$ & $\begin{array}{c}\text { Apparent Position }{ }^{\ddagger} \\
\text { J2000(RA, Dec) }\end{array}$ & $\begin{array}{c}\text { Selenographic Coordinates } \\
\text { (Lat., Lon.) }\end{array}$ \\
\hline $\begin{array}{c}\text { Santo Domingo } \\
\text { (The Dominican Republic) }\end{array}$ & 18.43567 & -69.96872 & 26 & 20.0 & $11.68 \pm 0.46$ & $(8.1826,20.2841)$ & $(-29.67,-67.81)$ \\
\hline $\begin{array}{c}\text { Georgia } \\
(\mathrm{USA})\end{array}$ & 32.51667 & -83.65440 & 107 & 16.0 & $10.76 \pm 0.44$ & $(8.1968,20.0198)$ & $(-29.56,-67.84)$ \\
\hline $\begin{array}{c}\text { Boa Vista } \\
\text { (Cape Verde, South Africa) }\end{array}$ & 16.14361 & -22.86400 & 55 & 16.0 & $10.70 \pm 0.47$ & $(8.1297,20.2223)$ & $(-29.51,-67.89)$ \\
\hline $\begin{array}{l}\text { Santa Cruz de Tenerife } \\
\text { (Cannary Island, Spain) }\end{array}$ & 28.14169 & -16.62200 & 1187 & 2.0 & $8.52 \pm 0.43$ & $(8.1291,19.9900)$ & $(-29.43,-67.84)$ \\
\hline $\begin{array}{c}\text { Karben } \\
\text { (Germany) }\end{array}$ & 50.21615 & 8.79607 & 140 & 1.0 & $8.54 \pm 0.49$ & $(8.1342,19.5625)$ & $(-29.29,-67.97)$ \\
\hline $\begin{array}{c}\text { Velky Osek } \\
\text { (Czech Republic) }\end{array}$ & 50.09820 & 15.18885 & 192 & 0.5 & $6.61 \pm 0.47$ & $(8.1334,19.5386)$ & $(-29.40,-67.94)$ \\
\hline $\begin{array}{c}\text { Vienna } \\
\text { (Austria) }\end{array}$ & 48.25000 & 16.21700 & 450 & 4.0 & $9.88 \pm 0.44$ & $(8.1315,19.5562)$ & $(-29.06,-67.99)$ \\
\hline
\end{tabular}

Table 2.1: Properties of the images involved in the analysis of L1-21-J.

site, see Section 2.4), is a short exposure (0.71 seconds) taken with the 25 " (635 mm) FL $=2700 \mathrm{~m}$ telescope of LaLoma Observatory in San Vicente Ferrer (Antioquia) in Colombia. The picture (see Figure 2.2) was taken using a $17.7 \times 13.4 \mathrm{~mm}(4656 \times 3520$ pixels $)$ CMOS ZWO ASI1600MC detector, working with a f/2.92 focal reducer in the primary focus of the telescope, yielding an efective $\mathrm{FL}=1854.2 \mathrm{~mm}$. Given the large aperture and low F-value of the telescope, the event was captured with a relatively short exposure. The picture was taken at 04:41:37 UTC, which also coincide with the time reported by MIDAS.

Each pixel of the camera attached to LaLoma observatory telescope, covers 0.32 arcsec, that in ideal atmospheric conditions correspond to a spatial resolution of $0.7 \mathrm{~km} / \mathrm{px}$ at the center of the Moon. At the location of the impact ( $\sim 60$ degrees from the center of the near side), the resolution will be larger than $2 \mathrm{~km} / \mathrm{px}$ (again, under idealized atmospheric conditions). However, since the actual seeing at the time of the picture was a few arcsec, the actual resolution downgrades to $\gtrsim 20 \mathrm{~km} / \mathrm{px}$.

The remaining lower resolution pictures contain background stars and they 


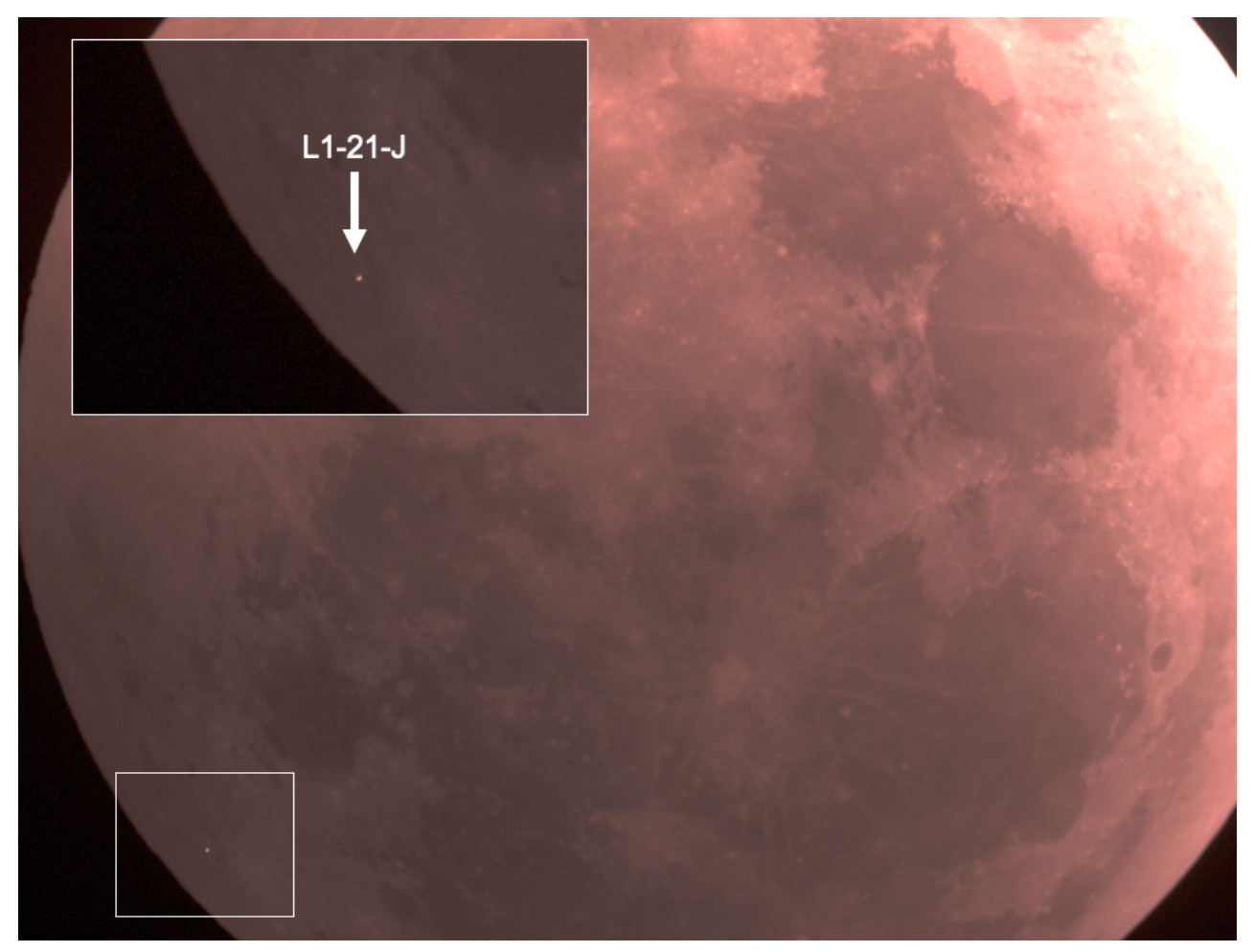

Figure 2.2: Picture of the total lunar eclipse at the time of the impact flash taken the observatory LaLoma, Colombia. Picture by Jonathan Ospina, Mauricio Gaviria and Sergio López. 
were used for a parallax-based (independent) estimation of the impact site (Section 2.4.2) and for the photometry of the flash (Section 2.6). For illustration purposes, we show In Figure 2.3, the picture taken by Fritz Pichardo in Santo Domingo, the Dominican Republic. The 20-seconds exposure, started at 04:41:24 UTC and lasted until 04:41:44 UTC including the time of the flash. The picture was taken using a Canon T3i DSLR camera (18Mpx APC-S CMOS sensor), installed on the secondary focus of an equatorially mounted 8 inch Celestron CPC 800 Schmidt-Cassegrain telescope, with a focal length $F L=2032 \mathrm{~mm}$ (f/10). The camera was installed with a focal reducer f/6.3, yielding an effective FL=1280.16 $\mathrm{mm}$.

Other pictures included a 16 seconds exposure taken by Petr Horálek in Boa Vista, Cape Verde. The image was taken using a Canon EOS 6D DSLR camera attached to a MTO 1100/f10.5. Gregory Hogan took a 16 seconds exposure from Kathleen, Georgia, USA using a Canon EOS 6D DSLR camera. Fritz Helmut Hemmerich captured the event with 2 seconds exposure time from Tenerife in the Canary Islands using a RASA 11"/F2.2, ASI 071 color camera (cooled to $-25^{\circ} \mathrm{C}$, at the highest dynamic range). From Velký Osek, Czech Republic, Libor Haspl took a 0.5 seconds exposure using a Canon 5D Mk IV mounted on a 8" telescope. From Vienna, Austria, Robert Eder Artis took 4 seconds exposure from his Canon 600Da astro modified DSLR camera with Skywatcher Newton 130/650 PDS. Lastly, we received from Dr. Sighard Schräbler and Dr. Ulrike Löffler from Karben, Germany a 1-second exposure taken with a Sony A7s DSLR camera mounted at the primary focus of 12 " Foto-Newton refelector.

For reproducibility purposes, we provide access to all the footage used in this work (raw images) in a companion GitHub repository. ${ }^{6}$

\footnotetext{
${ }^{6}$ https://github.com/seap-udea/MoonFlashes
} 


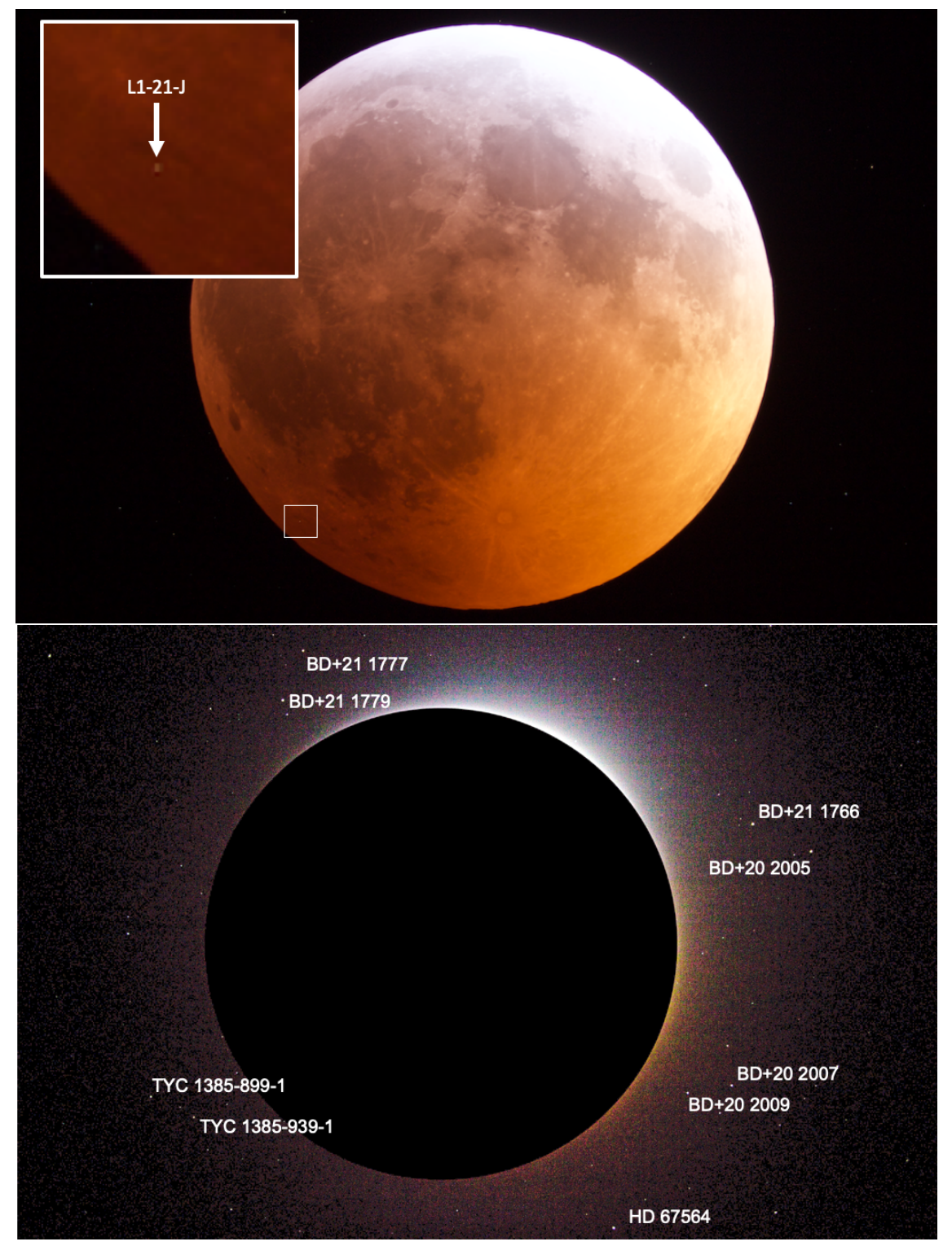

Figure 2.3: Picture of the total lunar eclipse at the time of the impact flash taken in Santo Domingo, the Dominican Republic. Upper panel: detail of the L1-21J flash. Lower panel: the same picture with the moon removed, highlighting the background stars. 9 stars were identified and used for the parallax and photometry analyses. Picture by Fritz Pichardo. 


\subsection{Location}

Determining the precise location of the impact from images that were not properly calibrated for this purpose can be challenging. Here we devise two independent procedures: a visual comparison between the highest resolution picture and LRO maps and a geometric-based location estimation.

\subsubsection{Visual procedure}

In Figure 2.5 we graphically summarize our visual procedure. We first superimpose and align our highest resolution picture (see Figure 2.2) with Lunar Reconnaissance Orbiter Camera (LROC) orthographic projection maps 7 of the southeast lunar quadrant. Then, we distort the flash image and superimpose it to a cylindrical equidistant projection of the selected region (lowest panel in Figure 2.5). This superposition allowed us to estimate the coordinates of the impact site and their corresponding errors.

According to our analysis, the impact happened to the southeast of Mare Humoris, near the easily identifiable Byrgius crater and inside a triangle with vertices in the Lagrange $\mathrm{H}, \mathrm{K}$ and $\mathrm{X}$ craters. The impact signature (crater and/or rays) should be inside an almost elliptical region centered at lat. -68.17, long. -29.43 with an east-west error of 18-km along the major axis, and a north-south error of $15 \mathrm{~km}$ along the minor axis. This is a reasonable-sized area, where prospecting satellites may look for a crater in the near future (see Section 2.7).

\subsubsection{Geometrical procedure}

In all pictures, 9 reference stars were identified (see for instance the lower panel of Figure 2.3). The position and brightness of these stars provide us valuable

\footnotetext{
7 https : //quickmap.Iroc.asu.edu
} 


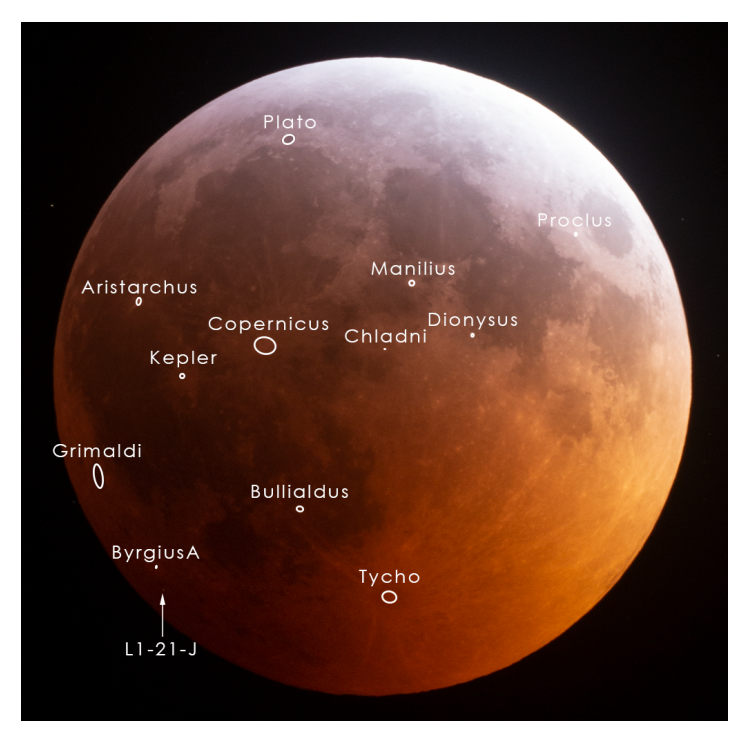

Figure 2.4: Craters and lunar features used by the Geometrical procedure in calibrating for the selenographic coordinates (section 2.4.2). Picture by Petr Horálek.

information for performing the astrometry and photometry on the images. In Tables 2.2 and 2.3 , we provide detailed information of all the stars identified in our images, including their sky coordinates, namely J2000 $(\alpha, \delta)$, image (centroid) coordinates, $(X, Y)$, magnitudes and counts on each image channel (see Section 2.6.).

Similarly, we identified and measure the position over all the images of 11 surface features (mainly large craters) as shown in Figure 2.4. In Table 2.4 we provide the selenographic latitude and longitude of these features, along with their calculated geocentric sky position and distance, as estimated with the procedure below.

Since our aim here is to estimate the selenographic location of the impact, we need a method to convert from image to selenographic coordinates. We perform this transformation in two steps. First, we calculate, for each image, the so- 


\begin{tabular}{|c|c|c|c|c|c|c|c|c|c|c|}
\hline \multirow[t]{2}{*}{ Star name } & \multicolumn{2}{|c|}{ Sky Position $^{\dagger}$} & \multicolumn{3}{|c|}{ Magnitudes } & \multicolumn{3}{|c|}{ Counts $\left(\mathrm{AP}^{\dagger \dagger}\right)$} & \multicolumn{2}{|c|}{ Coordinates } \\
\hline & $\mathrm{RA}$ & DEC & B & V & $\mathrm{G}^{\ddagger}$ & Red & Green & Blue & $\mathrm{X}$ & $\mathrm{Y}$ \\
\hline \multicolumn{11}{|c|}{$\mathrm{RD}$} \\
\hline $\mathrm{BD}+211766$ & 8.14149 & 20.59967 & 10.69 & 9.03 & 8.35 & 14161 & 6726 & 10608 & 4138 & 1119 \\
\hline BD+20 2007 & 8.14449 & 20.28416 & 10.52 & 9.82 & 9.79 & 6129 & 8166 & 5846 & 4020 & 2589 \\
\hline HD 67564 & 8.15532 & 20.11876 & 9.38 & 9.09 & 9.02 & 8175 & 13559 & 8294 & 3356 & 3389 \\
\hline TYC 1385-899-1 & 8.19466 & 20.30565 & 11.34 & 10.22 & 10.01 & 4051 & 4038 & 4235 & 752 & 2652 \\
\hline BD+21 1779 & 8.18160 & 20.77903 & 10.20 & 9.22 & 8.95 & 9724 & 9539 & 9033 & 1494 & 423 \\
\hline BD+21 1777 & 8.17964 & 20.83795 & 11.11 & 10.18 & 9.68 & 7304 & 4911 & 5530 & 1609 & 145 \\
\hline BD+20 2009 & 8.14832 & 20.27791 & 10.82 & 10.52 & 10.44 & 3494 & 6623 & 4223 & 3772 & 2630 \\
\hline BD+20 2005 & 8.13657 & 20.56272 & 10.35 & 9.04 & 8.63 & 11959 & 7866 & 9402 & 4467 & 1274 \\
\hline TYC 1385-939-1 & 8.19105 & 20.27807 & 12.78 & 10.66 & 10.35 & 4282 & 1768 & 2955 & 994 & 2768 \\
\hline L1-21J & $8.1826^{*}$ & $20.2841^{*}$ & - & - & - & 1621 & 1519 & 1619 & 1562 & 2712 \\
\hline \multicolumn{11}{|c|}{ Georgia } \\
\hline TYC 1385-116-1 & 8.17401 & 20.60905 & 11.56 & 10.81 & 10.58 & 42396 & 48328 & 43515 & 1416 & 1255 \\
\hline HD 68121 & 8.19736 & 19.96890 & 10.03 & 9.64 & 9.56 & 11381 & 158217 & 167910 & 3346 & 2285 \\
\hline TYC 1385-1052-1 & 8.17914 & 19.83440 & 11.55 & 10.26 & 10.01 & 71357 & 78914 & 60751 & 3769 & 1512 \\
\hline TYC 1385-368-1 & 8.16140 & 20.02100 & 12.30 & 11.71 & 10.94 & 30182 & 34984 & 31206 & 3213 & 742 \\
\hline TYC 1385-188-1 & 8.19596 & 20.50482 & 10.91 & 10.87 & 10.51 & 45118 & 68999 & 84704 & 1717 & 2198 \\
\hline TYC 1385-1391-1 & 8.19691 & 19.78950 & 12.80 & 11.54 & 11.06 & 26349 & 28871 & 24964 & 3893 & 2276 \\
\hline TYC 1385-1610-1 & 8.19571 & 19.64252 & 11.66 & 11.22 & 11.22 & 21978 & 30030 & 33100 & 4340 & 2233 \\
\hline TYC 1385-1675-1 & 8.20378 & 19.69782 & 11.78 & 10.60 & 9.89 & 66890 & 69991 & 55405 & 4166 & 2577 \\
\hline TYC 1385-376-1 & 8.20688 & 19.68792 & 12.17 & 11.28 & 11.39 & 18135 & 24503 & 26564 & 4194 & 2710 \\
\hline L1-21J & $8.1968^{*}$ & $20.0198^{*}$ & - & - & - & 42672 & 29831 & 19853 & 3192 & 2255 \\
\hline \multicolumn{11}{|c|}{ CapeVerde } \\
\hline $\mathrm{BD}+202005$ & 8.13657 & 20.56272 & 10.35 & 9.04 & 8.63 & 17763 & 18461 & 9372 & 2633 & 469 \\
\hline $\mathrm{BD}+211766$ & 8.14149 & 20.59967 & 10.69 & 9.03 & 8.35 & 16788 & 17315 & 9183 & 2697 & 251 \\
\hline BD+20 2007 & 8.14449 & 20.28416 & 10.52 & 9.82 & 9.79 & 7700 & 7629 & 4055 & 1776 & 320 \\
\hline TYC 13841851 & 8.09468 & 20.42690 & 10.93 & 10.06 & 9.90 & 7830 & 7740 & 4465 & 2604 & 2219 \\
\hline TYC 13843851 & 8.12374 & 20.08094 & 12.55 & 11.70 & 11.24 & 1944 & 1520 & 1097 & 1375 & 1271 \\
\hline TYC 13859851 & 8.14128 & 20.12704 & 10.57 & 10.13 & 10.04 & 6518 & 6032 & 3268 & 1356 & 542 \\
\hline BD+20 2009 & 8.14832 & 20.27791 & 10.82 & 10.52 & 10.44 & 4402 & 4829 & 2330 & 1726 & 171 \\
\hline TYC 13845091 & 8.11375 & 20.03512 & 11.79 & 11.63 & 11.50 & 1506 & 1586 & 814 & 1330 & 1697 \\
\hline HD 67150 & 8.12391 & 19.81766 & 8.27 & 7.69 & 7.54 & 56496 & 56205 & 30291 & 624 & 1424 \\
\hline L1-21J & $8.1297^{*}$ & $20.2223^{*}$ & - & - & - & 2708 & 2535 & 1816 & 1726 & 950 \\
\hline \multicolumn{11}{|c|}{ CanaryIslands } \\
\hline HD 67150 & 8.12391 & 19.81766 & 8.27 & 7.69 & 7.54 & 271150 & 348264 & 236429 & 3884 & 2146 \\
\hline HD 67424 & 8.14424 & 19.77072 & 8.53 & 8.48 & 8.47 & 93949 & 154985 & 139900 & 3825 & 2801 \\
\hline TYC 1385-985-1 & 8.14128 & 20.12704 & 10.57 & 10.13 & 10.04 & 30721 & 41212 & 29098 & 3069 & 2508 \\
\hline HD 67564 & 8.15532 & 20.11876 & 9.38 & 9.09 & 9.02 & 71428 & 99635 & 76508 & 2975 & 2946 \\
\hline $\mathrm{BD}+202007$ & 8.14449 & 20.28416 & 10.52 & 9.82 & 9.79 & 37012 & 47327 & 31202 & 2700 & 2519 \\
\hline BD+20 2009 & 8.14832 & 20.27791 & 10.82 & 10.52 & 10.44 & 19677 & 28174 & 21551 & 2683 & 2640 \\
\hline $\mathrm{BD}+202005$ & 8.13657 & 20.56272 & 10.35 & 9.04 & 8.63 & 108293 & 105234 & 47290 & 2152 & 2118 \\
\hline TYC 1384-185-1 & 8.09468 & 20.42690 & 10.93 & 10.06 & 9.90 & 33690 & 37133 & 21446 & 2780 & 906 \\
\hline TYC 1384-1748-1 & 8.10977 & 19.80182 & 11.72 & 11.26 & 11.13 & 9836 & 12076 & 8110 & 4030 & 1719 \\
\hline L1-21J & $8.1291^{*}$ & $19.9900^{*}$ & - & - & & 138565 & 94635 & 30931 & 3465 & 2210 \\
\hline \multicolumn{11}{|c|}{ Continues in Table 2.3} \\
\hline
\end{tabular}

Table 2.2: Reference stars properties, photometry and astrometry results for the pictures analysed in this work. 


\begin{tabular}{|c|c|c|c|c|c|c|c|c|c|c|}
\hline \multirow[t]{2}{*}{ Star name } & \multicolumn{2}{|c|}{ Sky Position $^{\dagger}$} & \multicolumn{3}{|c|}{ Magnitudes } & \multicolumn{3}{|c|}{ Counts $\left(\mathrm{AP}^{\dagger \dagger}\right)$} & \multicolumn{2}{|c|}{ Coordinates } \\
\hline & $\mathrm{RA}$ & DEC & B & $\mathrm{V}$ & $\mathrm{G}^{\ddagger}$ & Red & Green & Blue & $\mathrm{X}$ & $\mathrm{Y}$ \\
\hline \multicolumn{11}{|c|}{ Continued from Table 2.2 } \\
\hline \multicolumn{11}{|c|}{ Germany } \\
\hline HD 67424 & 8.14424 & 19.77072 & 8.53 & 8.48 & 8.47 & 2978 & 6118 & 5076 & 1358 & 950 \\
\hline HD 67346 & 8.13574 & 19.21594 & 8.23 & 7.63 & 7.48 & 5117 & 10885 & 6747 & 918 & 2278 \\
\hline TYC 1384-1330-1 & 8.09963 & 19.38749 & 11.00 & 10.05 & 9.76 & 704 & 1086 & 785 & 2221 & 2542 \\
\hline TYC 1384-594-1 & 8.08099 & 19.88256 & 10.66 & 9.87 & 9.61 & 744 & 1538 & 913 & 3395 & 1815 \\
\hline BD+20 1997 & 8.08191 & 20.09054 & 10.21 & 9.36 & 9.09 & 986 & 2104 & 1243 & 3628 & 1357 \\
\hline $\mathrm{BD}+202002$ & 8.11703 & 20.30866 & 10.83 & 10.37 & 10.20 & 464 & 969 & 671 & 2841 & 282 \\
\hline TYC 1385-985-1 & 8.14128 & 20.12704 & 10.57 & 10.13 & 10.04 & 557 & 1244 & 854 & 1890 & 247 \\
\hline TYC 1384-963-1 & 8.12454 & 19.45866 & 12.73 & 11.52 & 10.75 & 120 & 282 & 244 & 1562 & 1955 \\
\hline TYC 1385-1668-1 & 8.13691 & 19.31428 & 11.70 & 11.37 & 11.08 & 213 & 421 & 232 & 1008 & 2045 \\
\hline L1-21J & $8.1342^{*}$ & $19.5625^{*}$ & - & & & 3699 & 5351 & 2730 & 1400 & 1568 \\
\hline \multicolumn{11}{|c|}{ Czech } \\
\hline HD 67346 & 8.13574 & 19.21594 & 8.23 & 7.63 & 7.48 & 5081 & 6300 & 6616 & 1341 & 2032 \\
\hline HD 67424 & 8.14424 & 19.77072 & 8.53 & 8.48 & 8.47 & 3480 & 4637 & 6015 & 2871 & 992 \\
\hline TYC 1385-985-1 & 8.14128 & 20.12704 & 10.57 & 10.13 & 10.04 & 1215 & 1325 & 1218 & 3997 & 685 \\
\hline BD+20 2007 & 8.14449 & 20.28416 & 10.52 & 9.82 & 9.79 & 1366 & 1473 & 1508 & 4416 & 358 \\
\hline $\mathrm{BD}+202002$ & 8.11703 & 20.30866 & 10.83 & 10.37 & 10.20 & 951 & 1034 & 1041 & 4964 & 1493 \\
\hline HD 66551 & 8.08129 & 20.23307 & 9.07 & 8.93 & 8.91 & 2060 & 2469 & 3168 & 5358 & 3101 \\
\hline BD+20 1997 & 8.08191 & 20.09054 & 10.21 & 9.36 & 9.09 & 1650 & 1688 & 1666 & 4917 & 3250 \\
\hline TYC 1384-594-1 & 8.08099 & 19.88256 & 10.66 & 9.87 & 9.61 & 1113 & 1399 & 1142 & 4308 & 3544 \\
\hline TYC 1384-1330-1 & 8.09963 & 19.38749 & 11.00 & 10.05 & 9.76 & 1102 & 869 & 846 & 2488 & 3362 \\
\hline L1-21J & $8.1334^{*}$ & $19.5386^{*}$ & - & - & - & 6324 & 5347 & 3548 & 2360 & 1746 \\
\hline \multicolumn{11}{|c|}{ Vienna } \\
\hline HD 67346 & 8.13574 & 19.21594 & 8.23 & 7.63 & 7.48 & 31494 & 42730 & 25476 & 1570 & 2847 \\
\hline HD 67424 & 8.14424 & 19.77072 & 8.53 & 8.48 & 8.47 & 12565 & 19687 & 16399 & 1628 & 1493 \\
\hline TYC 13859851 & 8.14128 & 20.12704 & 10.57 & 10.13 & 10.04 & 2228 & 3462 & 2078 & 1939 & 695 \\
\hline BD+20 2007 & 8.14449 & 20.28416 & 10.52 & 9.82 & 9.79 & 3222 & 4585 & 2610 & 1929 & 304 \\
\hline TYC 13841851 & 8.09468 & 20.42690 & 10.93 & 10.06 & 9.90 & 3633 & 4292 & 2017 & 3635 & 389 \\
\hline BD+20 1997 & 8.08191 & 20.09054 & 10.21 & 9.36 & 9.09 & 7832 & 8560 & 4440 & 3851 & 1274 \\
\hline TYC 13845941 & 8.08099 & 19.88256 & 10.66 & 9.87 & 9.61 & 4928 & 5578 & 3010 & 3758 & 1762 \\
\hline TYC 138413301 & 8.09963 & 19.38749 & 11.00 & 10.05 & 9.76 & 3260 & 4414 & 2270 & 2855 & 2752 \\
\hline HD 67564 & 8.15532 & 20.11876 & 9.38 & 9.09 & 9.02 & 6683 & 10083 & 7036 & 1476 & 594 \\
\hline L1-21J & $8.1315^{*}$ & $19.5562^{*}$ & - & - & - & 8457 & 4877 & 2342 & 1921 & 2097 \\
\hline
\end{tabular}

Table 2.3: Continuation of Table 2.2. 


\begin{tabular}{cccc}
\hline \hline Surface Feature & $\begin{array}{c}\text { Selenographic Coordinates } \\
\text { (Lat.,Lon. })\end{array}$ & $\begin{array}{c}\text { Geocentric Position } \\
\text { J2000 }(\mathrm{RA}, \mathrm{Dec})\end{array}$ & $\begin{array}{c}\text { Distance }^{\dagger} \\
(\mathrm{km})\end{array}$ \\
\hline Moon Center & - & $(8.1667,20.4362)$ & 357046 \\
L1-21J & $(-29.42,-67.90)^{\ddagger}$ & $(8.1799,20.2505)$ & 356553 \\
Byrgius A & $(-24.54,-63.83)$ & $(8.1804,20.2709)$ & 356363 \\
Grimaldi & $(-5.53,-68.26)$ & $(8.1840,20.3505)$ & 356135 \\
Aristachus & $(23.69,-47.49)$ & $(8.1815,20.5024)$ & 355491 \\
Plato & $(51.64,-9.30)$ & $(8.1721,20.6433)$ & 355636 \\
Tycho & $(-43.40,-11.26)$ & $(8.1659,20.2433)$ & 356271 \\
Copernicus & $(9.64,-20.06)$ & $(8.1737,20.4634)$ & 354999 \\
Manilius & $(14.44,9.06)$ & $(8.1645,20.5177)$ & 355303 \\
Dionysus & $(2.77,17.29)$ & $(8.1608,20.4727)$ & 355576 \\
Chladni & $(3.47,-0.23)$ & $(8.1662,20.4603)$ & 355284 \\
Kepler & $(8.15,-37.99)$ & $(8.1789,20.4370)$ & 355312 \\
Bullialdus & $(-20.75,-22.30)$ & $(8.1715,20.3201)$ & 355721 \\
\hline \hline
\end{tabular}

$\dagger$ Calculated geocentric coordinates and distance (see Section 2.4.2

$\ddagger$ Calculated with our geometrical procedure (see Section 2.4.2

Table 2.4: Lunar features reference points and their selenographic coordinates, along with the apparent geocentric equatorial coordinate RA, Dec and geocentric distance results from parallax analysis. 
called plate constants, namely the coefficients of a linear (affine) transformation that convert image into sky coordinates and vice versa. The resulting projected sky position of the impact site, as computed with this procedure, are provided in Tables 2.2 and 2.3. We apply this transformation to compute also the projected position in the sky of the selected surface features.

Transforming selenographic into sky coordinates involves a complex rotation in the sky and the precise knowledge of the relative Earth-Moon position. In order to model the exact transformation required, we model a formula based on projecting latitude and longitudinal position on the spherical moon surface into an $\mathrm{X}-\mathrm{Y}$ coordinates. The $\mathrm{X}-\mathrm{Y}$ coordinate is then rotated and transformed by a general transformation matrix to convert it to RA-Dec. The resulting formula takes the form of:

$$
\begin{aligned}
\alpha= & a_{1} \sin \left(\lambda-\lambda_{0}\right) \cos \left(\phi-\phi_{0}\right) \\
& +a_{2} \sin \left(\phi-\phi_{0}\right)+a_{3} \\
\delta= & a_{4} \sin \left(\lambda-\lambda_{0}\right) \cos \left(\phi-\phi_{0}\right) \\
& +a_{5} \sin \left(\phi-\phi_{0}\right)+a_{6},
\end{aligned}
$$

where $a_{1}, \ldots, a_{6}$ are free coefficients and $\left(\phi_{0}, \lambda_{0}\right)$ are the unknown selenographic coordinates of the center of the moon (associated with lunar libration). Since we know the values of $\alpha, \delta, \lambda$ and $\phi$ of at least 11 points on the surface, we can find the best-fit values for the 8 free parameters of this general transformation. Once we have the parameters of the selenographic to sky coordinates transformation, we may invert it to estimate the position of the impact, according to the information available on each image.

Combining the location obtained after applying this geometrical procedure to all the images, we estimate that the impact happened inside an "ellipse" having 
selenographic coordinate centered around lat. $-29.43_{-0.21}^{+0.30}$ and lon. $-67.89_{-0.09}^{+0.07}$. This result is in fair agreement with our visual estimation.

In the lowest panel of Figure 2.5, we show the position and error areas corresponding to our visual and geometrical procedures. Interestingly, the resulting ellipse from the geometric method span larger along the latitudinal direction as oppose to the longitudinal direction. The projection of the apparent coordinate onto the selenographic surface would suggest a larger error along the longitudinal direction. However, the statistical process has put a tighter constraint along this direction instead.

\section{Lunar parallax}

In addition to tangential position along the surface of the moon measured in section 2.4, we can also measure the radial distance using lunar parallax. The idea of using lunar parallax to measure the distance to the Moon has been proposed since the time of the Ancient Greece. However, to put this into practice with high precision is often complicated by practicality. First the high dynamic range between the Moon and background stars makes it difficult to get both resolved in a single exposure. It requires coordination between multiple observers separated by vast geographical distances to be making observation simultaneously. More importantly, it requires meticulous synchronization in the observation time among all observers.

The total lunar eclipse offer the perfect set of circumstances for lunar parallax measurements. It is an event that is observed simultaneously worldwide across vast geographical distances. The moon brightness is dimmed enough that background stars can be easily resolved. Most importantly, the meteor flash on the lunar surface guarantees that the exposures overlap to the same fractions 

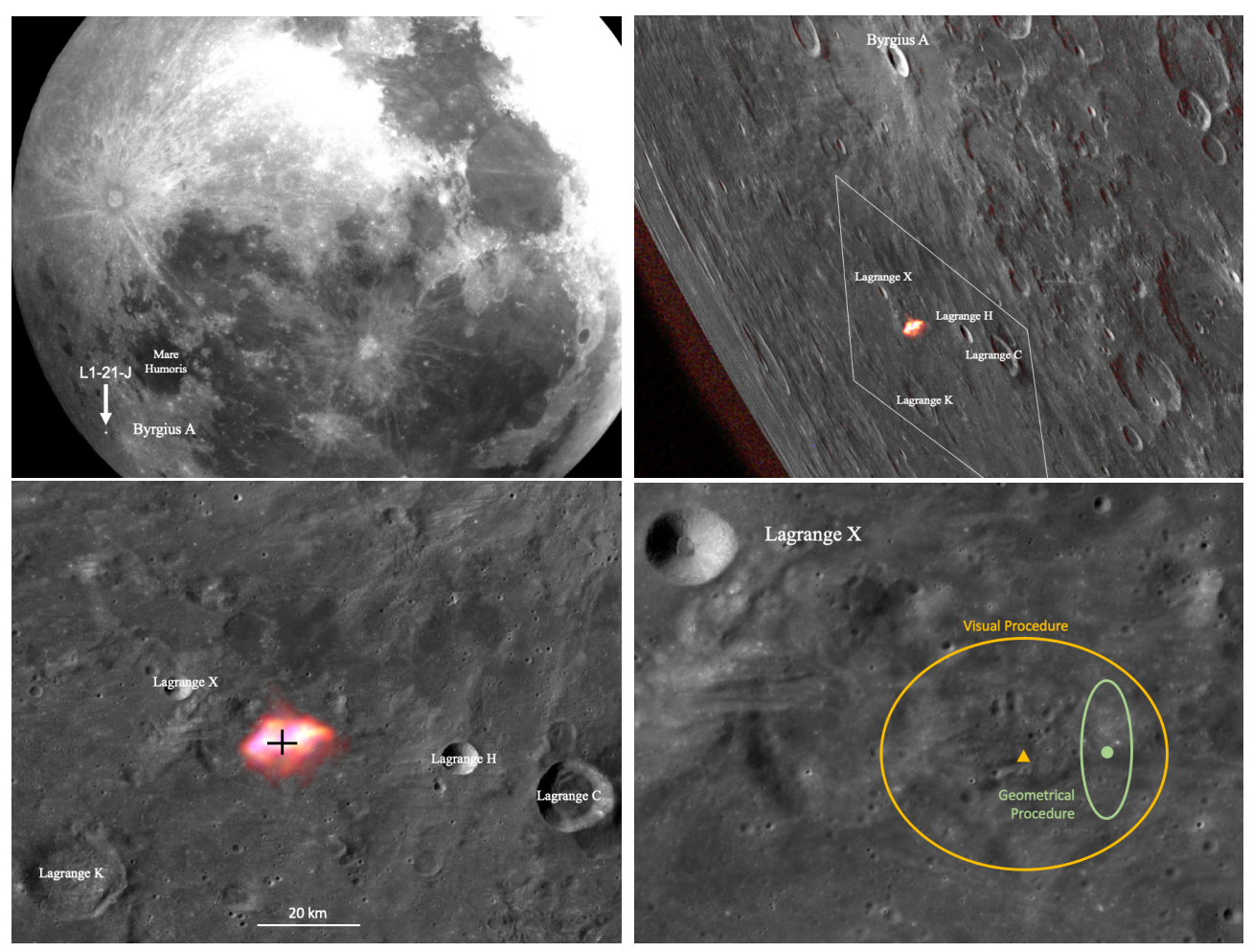

Figure 2.5: Impact estimated location. Upper row: original picture taken at LaLoma observatory in Colombia (left) and superposition of the original flash image and a LROC ortographic map (right). Bottom row: image of the impact flash superimposed to a high resolution LROC cylindrical map (left) and error regions of the visual and geometrical procedures (right).

of a second during which the impact occurred at 04:41:37 UTC on January 21, 2019.

For each image, the apparent coordinate $\left(\alpha^{\prime}, \delta^{\prime}\right)$ of the flash and center of the Moon is measured (Table 2.1). The geocentric parallax correction is then calculated for each observer location. The geocentric (common) sky coordinates $(\alpha, \delta)$ of the impact site are related to their apparent values by [23]: 


$$
\begin{gathered}
\tan \left(\alpha-\alpha^{\prime}\right)=\frac{\rho \cos \phi^{\prime} \sin H}{r \cos \delta-\rho \cos \phi^{\prime} \cos H} \\
\tan \delta^{\prime}=\cos H^{\prime}\left(\frac{r \sin \delta-\rho \sin \phi^{\prime}}{r \cos \delta \cos H-\rho \cos \phi^{\prime}}\right),
\end{gathered}
$$

where $r$ is the geocentric distance in units of the (mean) Earth radius (6378.1366 $\mathrm{km}), \rho$ is the distance of the observer from the center of the Earth, $\phi$ is the geocentric latitude, $H$ is the geocentric hour-angle, and $H^{\prime}$ is the apparent hour-angle $H^{\prime}=H+\alpha^{\prime}-\alpha$. The Earth profile is approximated to be an oblate spheroid with flattening ratio of $1: 298.2575$.

Comparing the apparent coordinates of the flash (or any other point on the surface of the moon) as measured from different observing sites, with those calculated with eq. 2.4.4 - 2.4.5), it is possible to fit the value of $r, \alpha$ and $\delta$.

Using this procedure, we find that the geocentric coordinate of the impact site was $(\alpha, \delta)=(8.17992,20.25050)$ and its distance at the precise time of the event was $356553 \mathrm{~km}$. A similar procedure was performed for the center of the moon, finding $(\alpha, \delta)_{\text {center }}=(8.16674,20.43615)$ and a distance of $d_{\text {center }}=357046$ $\mathrm{km}$. For comparison NASA NAIF/SPICE $\mathrm{E}^{8}$ predicts for the center of the moon $(\alpha, \delta)_{\text {center }}^{\text {theo }}=(8.16604,20.43654)$ and a distance $d_{\text {center }}^{\text {theo }}=357745 \mathrm{~km}$. The difference between the theoretical predictions and those obtained with our procedure are within the errors expected for these quantities given the quality of our data.

With this additional information, we can provide an alternative method to calculate the selenographic coordinate of the impact using geocentric (common) sky coordinates $(\alpha, \delta)$. With the parallax measurement of 11 lunar surface features shown in table 2.4. we are able to construct the apparent geocentric equatorial coordinates to those features and L1-21-J. From these apparent geocentric equatorial coordinate, we can perform the least square fit to find the transforma-

\footnotetext{
$\sqrt[8]{\text { https://naif.jpl.nasa.gov/naif/ }}$
} 
tion coefficients to transform the coordinates back to selenographic coordinates using eq. 2.4.2)-2.4.3 in Section 2.4.2. From these transformation coefficients, the selenographic coordinate of L1-21-J can be calculated from geocentric (RA,Dec) to be at selenographic lat. $-29.42 \pm 0.20$ and lon. $-67.90 \pm 0.08$.

In this parallax measurement, angle measurement remains the largest source of error. The error source from GPS in determining the observer's location is virtually negligible compared to the astrometric errors, even when assuming uncertainties in the GPS as high as $100 \mathrm{~m}$. In all measurements we are able to obtain order of arcseconds in precisions. With the median tangential distance between observers around $2000 \mathrm{~km}$ and the lunar parallax angle around 0.6 degrees between two observations, this translate to the error in distance measurements in the order of $\pm 200 \mathrm{~km}$, which is equivalent to the relative error in lunar parallax distance of $0.05 \%$.

The precision provided by this method is enough for the distances between different selenographic coordinates to be visible (see Table 2.4). The diurnal libration is noticeable among images that results in differing amount of parallax between the center and near the limb of the moon surface. This resulted in parallax distances that are different among different position along the lunar surface, with selenographic coordinate closer to the center having shorter distance. These variation in distances among different selenographic coordinates is consistent with subtracting Earth-Moon distance with spherical projection on the surface of the moon with radius $R_{\text {moon }}=1,737.5 \mathrm{~km}$. This is comparable to having a depth perception that allow for the curvature of the moon to be perceived via parallax. In fact, by using a cross-eyed technique on a pair of lunar images in Figure 2.6 one can easily see the eclipsing moon in 3D with his or her own eyes. 


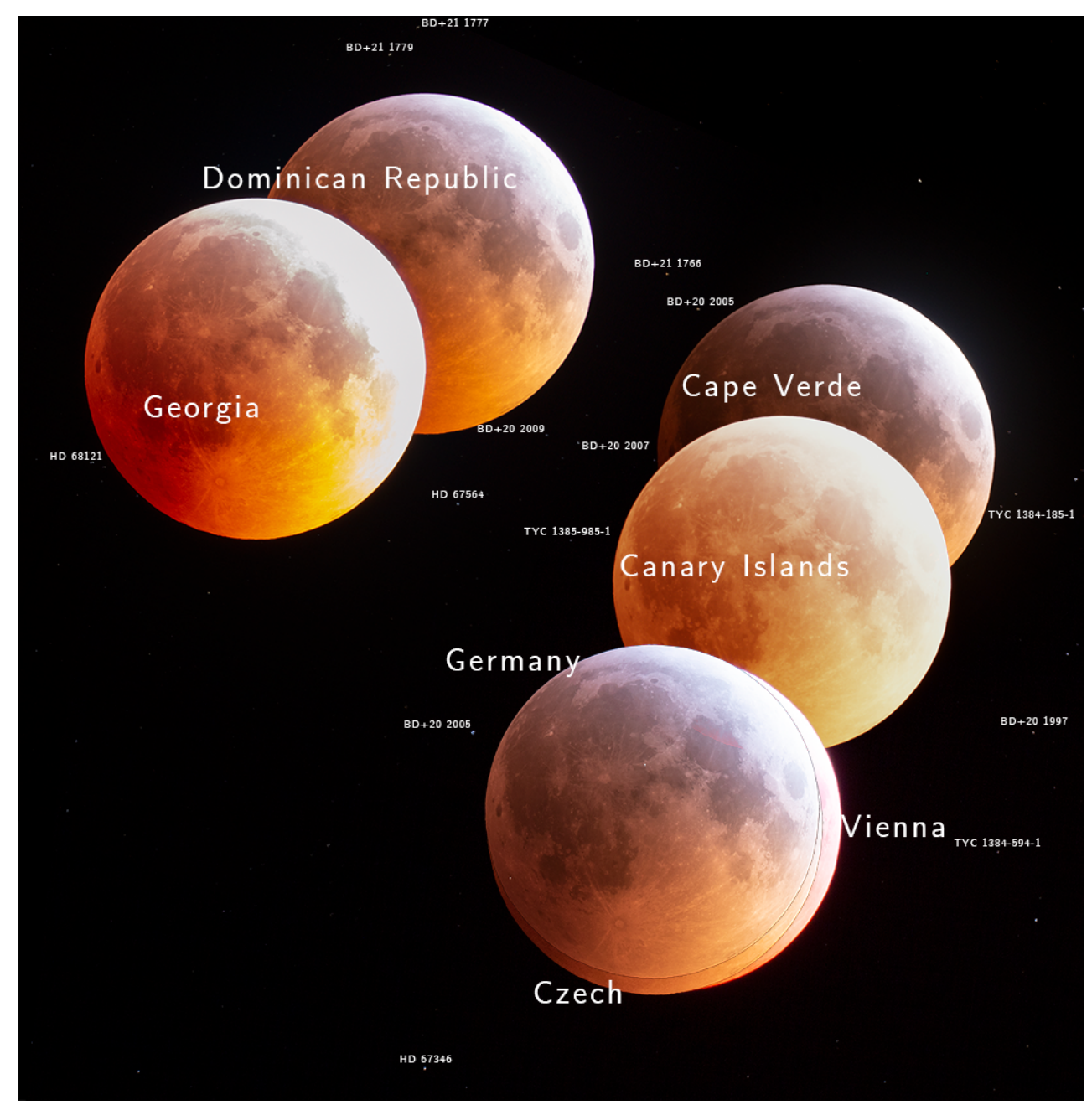

Figure 2.6: Overlay of all the apparent lunar position as observed by different observers. The angle differences between each images are used in Parallax calculations (section 2.4.2). All the images are rearranged so that the background stars are alligned. Some reference stars used in photometry are displayed. The apparent positions trace a rough geographical location of the observers, with observations from Europe stacked on the bottom right, the Macaronesian (East Atlantic) islands on the right, with Continenal US and the Caribbean on the top left. 


\subsection{Orbit}

It is impossible to reconstruct the complete orbit of a lunar impactor using only the observation of one single flash. Still, we can use the time and location of the impact to constraint the incoming direction and speed of the object.

For this purpose, we apply Gravitational Ray Tracing (GRT), a novel numerical technique recently introduced by [24] with the aim of computing efficiently the meteoroid impact probabilities on the surface of any planetary body in the Solar System. The method was tested on Earth and it successfully reproduced the impact speed distribution of moderately large meteoroids impacting our atmosphere.

In the GRT, random incoming directions (elevations and azimuths) are generated following a blue-noise distribution on the sky above the impact site (see [24] for a detailed explanation). These random directions are combined with a set of regularly spaced impact speeds in the interval between the moon escape velocity and the escape velocity from the Solar System at the distance of the Earth-Moon system to build many different random initial conditions. For each initial condition, the trajectory of a test particle is integrated backwards in time in the gravitational field of the Solar System. After one year, the heliocentric orbital elements of the test particle (namely its asymptotic orbit) is compared against the orbital elements of the population of already discovered Near Earth Objects (NEOs).

The (relative) probability that the actual meteoroid comes from one of the many directions and impact speeds in the simulation, is proportional to the density of NEOs in the space of classical orbital elements, around the asymptotic orbit. In other words, a given initial condition is more probable if many potential parent objects in the NEOs population have orbital elements similar to that of 
the asymptotic orbit associated to that condition.

Further details of the technique are found on the original paper by Zuluaga et al. [24].

For our purpose here, we generate 997 random incoming directions on the sky above the impact location, with a minimum separation of $5^{\circ}$ (incoming directions are not random, but carefully arranged to have a minimum separation with its closest neighbors; this configuration is intended to avoid numerical artifacts arising from the sampling procedure). Finally, we choose 100 regularly spaced impact speeds, that together with the incoming directions, create a set of 49901 different initial conditions.

41167 test particles (82\%) survived the numerical experiment (they did not collide against the Moon, the Earth or the Sun, nor escaped from the Solar System after being perturbed by the planets).

For each particle ("ray"), a (relative) probability, proportional to the density of NEOs around the asymptotic orbit, was computed. Using this (relative) probability, we can now estimate the marginal probability distributions (mpd) of any dynamical or spatial property associated to the trajectory of the meteoroid.

Thus, for instance, the probability that the impact speed was between $v$ and $v+\Delta v$ is simply the sum of the (relative) probabilities of all initial conditions having incoming speeds in this interval. The same method can be used to compute the mpd of the impact angle or radiant position.

In Figure 2.7 we show the ppds of the impact speed and incident angle (elevation) at the time and location of L1-21J event.

Interestingly the impact speed, $v_{\text {imp }}=13.8_{-4.3}^{+7.3} \mathrm{~km} / \mathrm{s}$ is lower than the typical values assumed for this quantity $(16-22 \mathrm{~km} / \mathrm{s}$, [8, 14]). Zuluaga[24] already shown that impact speeds may be different depending on the geographic location on the planetary surface (see Figure 11 of their paper). Additionally, most of 

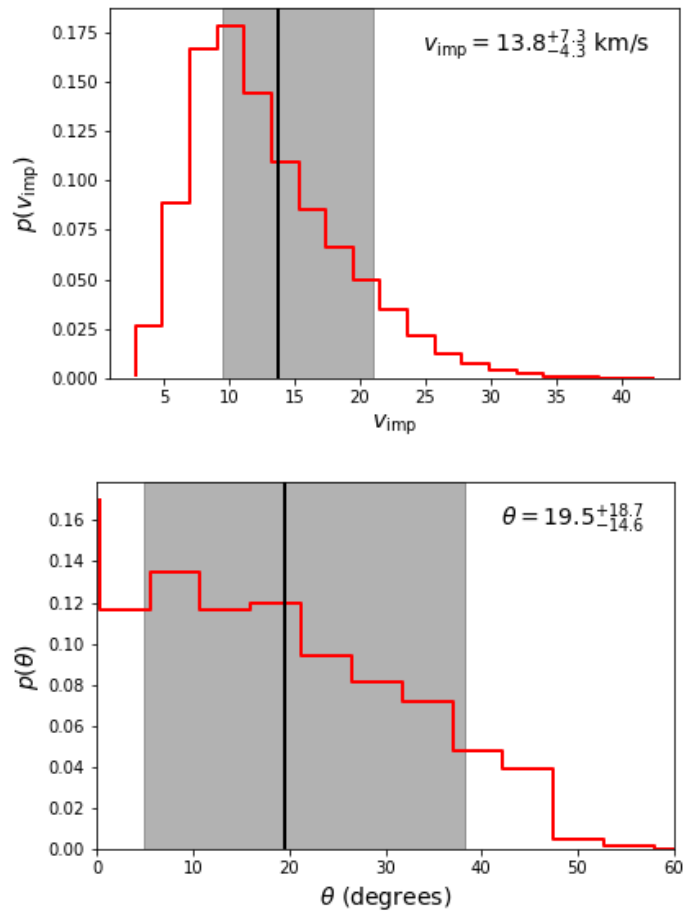

Figure 2.7: Marginal probability distributions (mpd) of Impact speed (upper panel) and incident angle (lower panel), as computed using a GRT analysis at the time and location of L1-21J event.

the NEOs used in the GRT analysis, are asteroids with low relative velocity to Earth.

The speed of sporadic cometary meteoroids and that of particles coming from major meteor shower are significantly larger than this. Still, in the absence of any particular clue about the nature of the object, assuming that it originates among more abundant objects is a reasonable assumption.

The incident angle distribution is biased towards shallow, $\lesssim 38.2$ degrees, impact directions. An almost vertical impact has an almost null probability. This effect, is a combination of dynamical factors and the fact that the distribution of NEOs is concentrated around the ecliptic plane. Since the impact site is at 

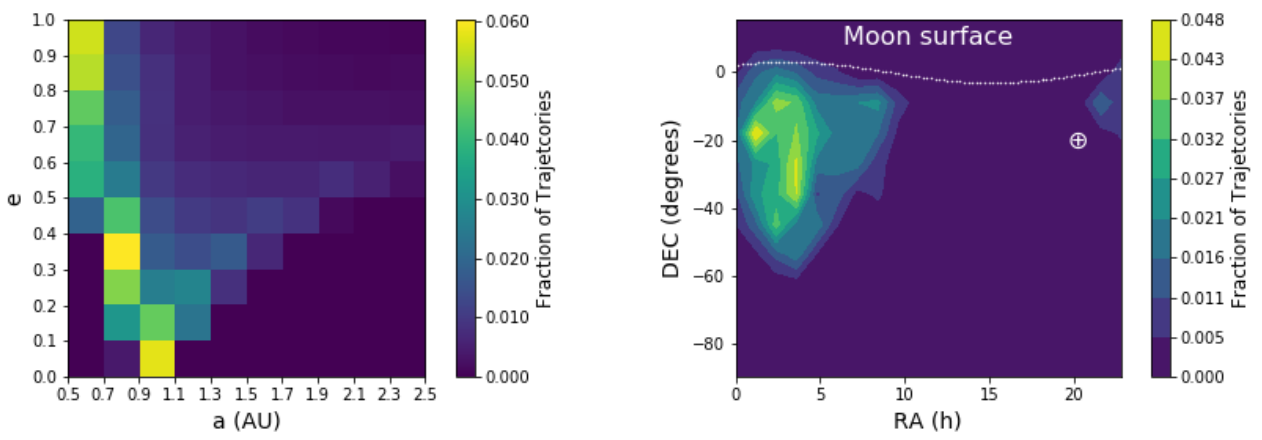

Figure 2.8: Heat maps of the two-dimensional marginal probability distribution of orbital elements of the parent body (left panel) and radiant locations on the sky (right panel). The dotted line in the right panel represent the boundary between the sky and the solid moon at the impact site. The Sun (and the Earth during the total lunar eclipse) are located in the sky above the impact site close to the $\oplus$ symbol.

low latitude, vertical incidence would be associated to inclined asymptotic orbits which are less common among NEOs.

Finally, we calculate the two-dimensional marginal probability distribution of the orbital elements of the parent body and its radiant on the sky above the impact site. The results are shown in Figure 2.8.

The distribution of orbital elements seems to favor the hypothesis that the impactor came from an orbit inside the Earth's orbit. If asteroidal in origin, its parent body could be probably classified as an Atens.

Regarding its incoming direction in the sky, our simulation seems to favor a extensive region around $\mathrm{RA} \sim 3.1 \mathrm{~h}, \mathrm{DEC} \sim-23.7$. No major meteor shower has a radiant around those positions. 


\subsection{Energy of the impact}

Flashes are the result of thermal emission from hot plasma plumes created by vaporized material coming from the meteoroid and the surface [25]. The impact event takes place in a very short time, ie. $\sim 10^{-2} \mathrm{~s}$, but visible light-emission last

for $\gtrsim 0.05-0.1 \mathrm{~s}$. In order to estimate the energy of the impact it is necessary to measure the brightness and duration of the flash.

\subsubsection{Photometry}

To illustrate the method we use for estimating the average flash brightness, as a reference image we use the picture taken by Fritz Pichardo in the Dominican Republic (see Figure 2.3). In the picture, the flash was detected at a SNR $\sim 10$. At least 9 well-known stars were also identified with similar SNR.

In Table 2.2 and 2.3 we show the properties of the reference stars, along with the value of the counts detected around their position (as determined with aperture photometry, AP) in each image channel (RGB). For completeness, we also include the counts detected on the flash position.

Performing precision photometry with RGB images is challenging. Although the spectral response of the camera sensor is well-known [26] and it covers all the visible spectra in a similar fashion that standard photometric filters, understanding how to relate the counts in each channel to a magnitude in a specific photometric system is not trivial.

We assume for simplicity that the Gaia G magnitude (which is already known for all the reference stars and also covers a wide region of the visible spectrum) could be in principle calculated from the counts in the RGB channels $C_{R}, C_{G}$ and $C_{B}$, using the formula: 


$$
G=Z+k_{R} \log C_{R}+k_{G} \log C_{G}+k_{B} \log C_{B}
$$

Here $Z$ is the unknown zero-point and $k_{R}, k_{G}$ and $k_{B}$ are also unknown constants.

The value of these constants were found by fitting with the previous formula the $\mathrm{G}$ magnitude of the reference stars on each image.

In Table 2.1 we present the G magnitude of the flash as measured after performing the AP photometry of our seven images, along with their corresponding errors.

Since exposure time for the reference stars in each image is larger than the duration of the flash $\left(t_{f}=0.3 \mathrm{~s}\right.$ ), The magnitude $G(t)$ (with $t$ the exposuretime) estimated with this procedure will under- or over-estimate the actual, $G_{f}$ average magnitude of the flash. $G(t)$ and $G_{f}$ will be related by the Pogson's law:

$$
G(t)=G_{f}-2.5 \log \left(t / t_{f}\right)
$$

Therefore, if we have independent $G(t)$ values, the true-average magnitude of the flash $G_{f}$ can be estimated by finding the intercept of the best-fit line in Eq. 2.6 .7 with the $\log t_{f}$ vertical line. In Figure 2.9 we show the result of applying this procedure to our observations of the L1-21J event.

We find that combining all this data the flash magnitude is:

$$
G_{f}=6.7 \pm 0.3
$$

where the error arises from the dispersion of the $G$ magnitudes estimated for different images.

We attempted to measure the relative brightness profile of the flash using the timeandddate video. For that purpose we performed aperture photometry 


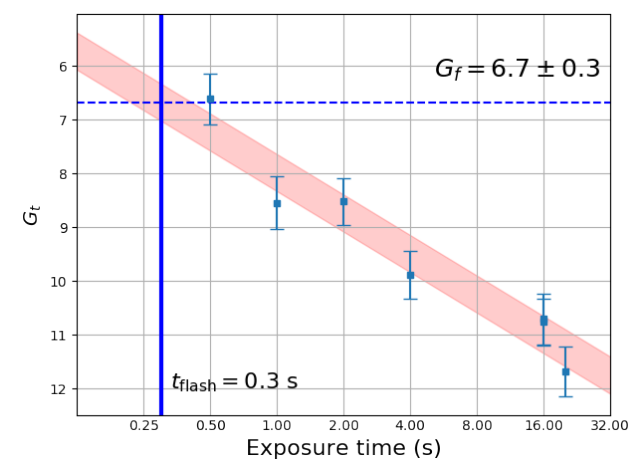

Figure 2.9: Apparent $G^{\prime}$ magnitude as observed by each image with varying exposure time. The intercept at the flash duration $t=0.3 \mathrm{~s}$ provide the estimation of the true flash magnitude.

on the flash image on each frame. We find that, for the sensitivity and time resolution of the video camera, no significant variation in the brightness was detected during the 0.3 seconds of the flash.

\subsubsection{Luminous and kinetic energy}

From the estimated magnitude in the G filter, we can estimate the visible luminous energy of the impact [20]:

$$
E_{r}=b_{G} 10^{\left(-G_{f}+G_{0}\right) / 2.5} \Delta t \Delta \lambda_{G} f \pi R^{2},
$$

where $R=357745 \mathrm{~km}$ is the distance to the Moon at the time of the impact, $b_{G}=2.5 \times 10^{-} 11 \mathrm{~W} / \mathrm{m}^{2} / \mathrm{nm}, G_{0}=0.03$ and $\Delta \lambda_{G}=420.360 \mathrm{~nm}$ are the calibration properties of the filter $9 t$ is the flash duration, and $f$ is the degree of anisotropy of the light emission (if the light was emitted isotropically from the surface then $f=2$. Conversely, if light is emitted at a very high altitude then $f=4$, see eg. [20]).

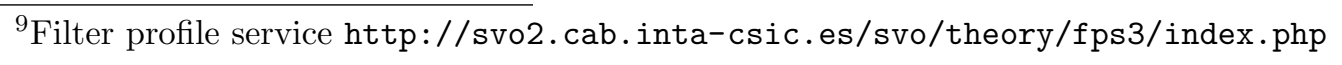


Using the estimated (average) flash magnitude $G_{f}=6.7 \pm 0.3$ and assuming $f=2$, we may finally estimate the total luminous energy released during the flash:

$$
\log \left(\frac{E_{r}}{\mathrm{~J}}\right)=6.7 \pm 0.349
$$

Estimating the kinetic energy of the meteoroid from the luminous energy emitted by the impact plume is tricky. We previously mentioned (Eq. 2.2.1) that it is customary to assume that both quantities differs only by a multiplicative "constant", namely the luminous efficiency $\eta$. Although this is an oversimplification of a very complex process, Rubio[25] achieved at fitting the flux of the leonids using a luminous efficiency of $\eta=2 \times 10^{-3}$. Independently Ortiz[16] and Madiedo [19, 20] used the same method to obtain efficiencies in the range of $0.0018-0.0034$ for different meteor showers.

If we assume that $\log \eta=-2.61 \pm 0.14$, the kinetic energy for the L1-21J impact will be:

$$
\log \left(\frac{K}{\mathrm{~J}}\right)=9.35 \pm 0.38
$$

This is equivalent to the explosion of $0.2-1.3$ tons of TNT.

\subsection{Impactor and crater size}

Once kinetic energy is calculated we may estimate the physical properties of the meteoroid and the crater size left by the impact.

The mass of the meteoroid can be calculated from the kinetic energy definition:

$$
M=2 K / v_{\mathrm{imp}}^{2}
$$


From there and assuming a proper bulk density $\rho$, we may also compute the object diameter:

$$
D=2\left(\frac{3 M}{4 \pi \rho}\right)^{1 / 3}
$$

The size of the crater, on the other hand, can be estimated using the wellknown scaling-relationship [27, 28]:

$$
d=0.25 \rho^{1 / 6} \rho_{t}^{-0.5} K^{0.29}(\sin \theta)^{1 / 3} .
$$

where $\mathrm{d}$ is the crater diameter and $\rho_{t}=1600 \mathrm{~kg} \mathrm{~m}^{-3}$ is the moon (regolith) surface density [20].

Besides $K$, the estimation of $M, D$ and $d$, requires educated guesses for the unknown properties $v_{\text {imp }}, \theta$ and $\rho$.

In previous works it was customary to assume typical impact velocity in the range of 16-20 km/s for sporadic meteors [10, 9] or $\sim 40-72 \mathrm{~km} / \mathrm{s}$ for specific meteor showers [14, 20]. The value of $\theta$ was always guessed in the absence of observational evidence able to constrain it.

Here, our dynamical model provide us marginal probability distribution function for these quantities (see Figure 2.7). Thus, instead of replacing the value of educated guesses, we can compute posterior probabilities distributions (ppd) for the desired quantities.

For this purpose we perform a simple Monte Carlo simulation where 1,000 Values of $\log K$ and the uncertain parameters $v_{\text {imp }}, \theta$ and $\rho$ were generated according to their marginal probability distribution. The values of $\log K$ was generated assuming a gaussian distribution of mean and standard deviation given by Eq. 2.6.11. $v_{\text {imp }}$ and $\theta$ where independently generated using the distributions computed in Section 2.5. 
The case of $\rho$ is interesting. The density of typical meteoroids impacting the Moon ranges from $1000 \mathrm{~kg} / \mathrm{m}^{3}$ (in the case of soft cometary materials) to 3700 $\mathrm{kg} / \mathrm{m}^{3}$, the density of ordinary chondrites ([14] and references there in). Since we do not know the nature of the impactor, we should assume several values for the densitiy.

For our Monte Carlo we use the following values for the density: $\rho_{1}=1000$ $\mathrm{kg} / \mathrm{m}^{3}$ with a probability of $1.0 \%$ (approximately matching the fraction of NEOs which are comets ${ }^{10}$ ), $\rho_{2}=2500 \mathrm{~kg} / \mathrm{m}^{3}$ with a probability of $59 \%$ (arising from parent bodies with Tisserand parameters below 2, [29]) and $\rho_{3}=3700 \mathrm{~kg} / \mathrm{m}^{3}$ with a probability of $40 \%$ (arising from parent bodies with large Tisserand parameters, [29]).

In Figure 2.10 we show posterior probability distributions for meteoroid mass $M$, diameter $D$ and crater size $d$.

We find that, in order to explain all the observations, the meteoroid producing the L1-21J impact should be roughly around a "football" sized $(18-41 \mathrm{~cm})$. Depending on its density the mass of the object could be in the range 7-79 kg. Given the shallow angle the crater diameter will be in the range $5-12 \mathrm{~m}$. This is well within the best resolution of the lunar prospecting moon satellites.

\subsection{Discussion}

These conclusions and values of the key quantities published in this work should not represent the final word about this historical phenomenon. This is only one of the first approximation to understanding the conditions on which the impact takes place.

Still, our work is original in two particular aspects: 1) it combines hetero-

\footnotetext{
$1 0 \longdiv { \text { https://cneos.jpl.nasa.gov/stats/totals.htm } }$
} 

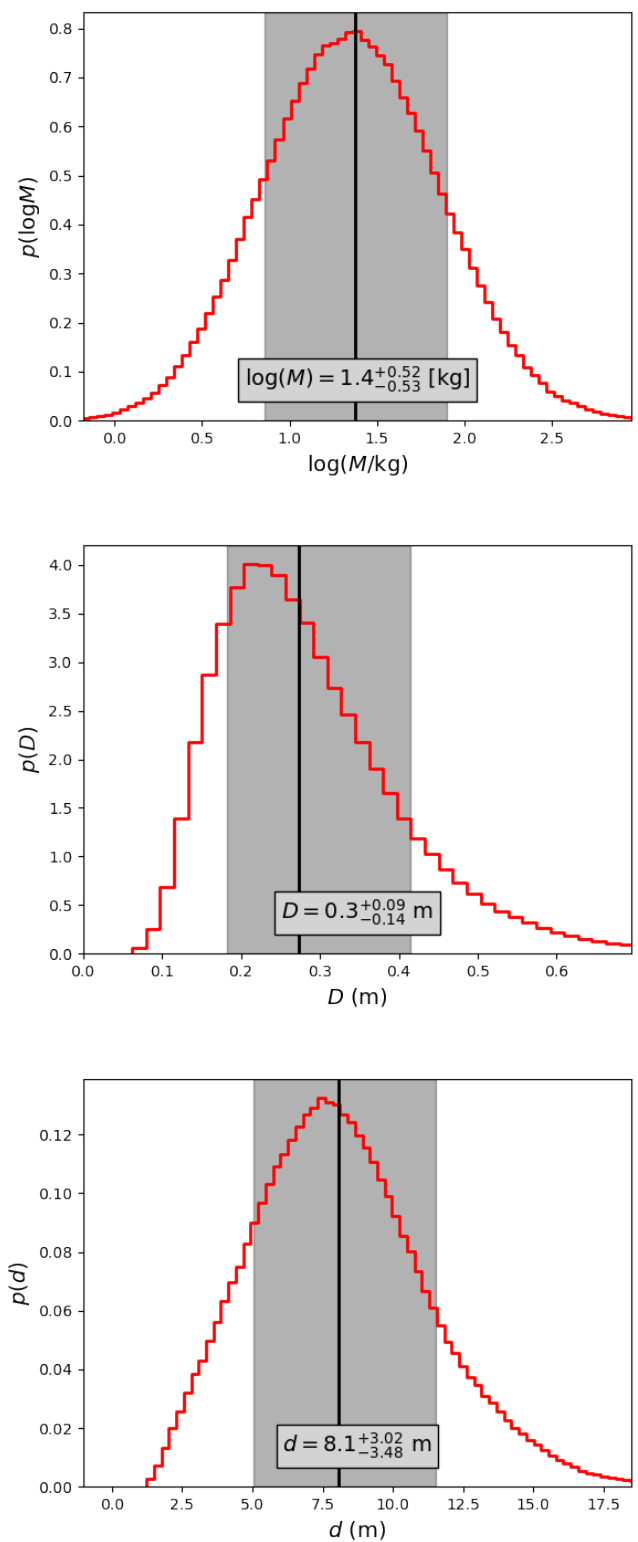

Figure 2.10: From top to bottom, posterior probability distributions (ppd) of meteoroid mass, diameter and crater size. 
geneous data obtained by amateur astronomers, to produce an "homogeneous" scientific analysis of the phenomenon. 2) instead of guessing some of the key quantities involved in the impact analysis, our work apply a numerical technique (the GRT) to compute the probability distribution of those quantities. Although the errors in the resulting quantities (energy, meteoroid and crater size) seems to be larger than usual, they are better supported statistically.

Our estimation of the impact location suffers from several systematic and random errors. Location determined by visual inspection of pictures and lunar maps is prone to conscious and unconscious biases. A geometrical method could potentially avoid human bias. However, since the flash happened close to the Moon limb, small error in a geometrical procedure may produce final errors even larger than those obtained with the visual method. Fortunately, it is not the case here.

We do not have any information about the velocity and the angle on which the object impact the surface of the Moon. The results published here rely on a novel numerical and statistical procedure that still needs to be tested in a wider diversity of conditions.

The GRT method relies on knowing precisely the distribution of NEOs in the space of orbital elements. However, the distribution of these objects is incomplete below $\sim 50 \mathrm{~m}$ and down to the estimated size of the impactor $(\sim 10 \mathrm{~cm})$. Still, assuming that very small objects follow closely the distribution of their larger parent (or cousin) objects, is not bad as a starting point.

The future discovery of the crater left by the impact (if resolvable) and its comparison with the predictions of this model, will greatly contribute to improve it and to test the validity of the GRT technique.

The success of the GRT technique will not only confirm the result provided by this work, but can also serve as a foundation to which future work in similar 
areas can progress. In the future, technique similar to GRT could be applied to observations on other solar system objects such as impacts on Jupiter or its moon.

The special set of circumstances that lead to this event have been quite rare. However, it is conceivable that with increasing interest in astronomy and availability of digital cameras another impact on the moon during the future lunar eclipses might again be observed simultaneously among many observers. This research should be able to set as a framework to the level of analysis that can be obtained from future observations made by amateur and professional astronomers alike.

Our work was the result of a timely collaboration between professional and amateur astronomers. The well-known skills of amateurs to collect and process high quality data of astronomical events, together with the capacity of professionals to convert this data into scientific results, is becoming a powerful driver of new scientific discoveries in astronomy.

\section{Acknowledgements}

We want to thank all people in social networks that share their pictures and data and that finally allow professional and amateur astronomers to work on this. We are particularly grateful to the MIDAS survey for discovering the impact and announcing early (without any embargo). This generosity allow all of us to discover the data hidden in pictures and videos. Finally we especially want to thank the careful review of the manuscript made by Dr. Rodrigo Leiva from Southwest Research Institute, his suggestions and comments were very important for our work. We would also like to thank all the amateur astronomers that contributed the images necessary for this work: Petr Horálek from Cape 
Verde, Gregory Hogan from USA, Fritz Helmut Hemmerich from Canary Islands, Libor Haspl from Czech Republic, Robert Eder Artis from Austria, Dr. Sighard Schräbler and Dr. Ulrike Löffler from Germany, and a couple more amateur astronomers who provided their images, but were unable to meet the scientific precision required. 


\title{
Chapter 3
}

\section{Testing the Weak Equivalence Principle with Cosmological Gamma Ray Bursts ${ }^{1}$}

\begin{abstract}
Gamma Ray Bursts (GRBs) with rapid variations at cosmological distances are used to place new limits on violations of the gravitational weak equivalence principle (WEP). These limits track intrinsic timing deviations between GRB photons of different energies as they cross the universe, in particular in the $\mathrm{KeV}$ to GeV energy range. Previous limits in this energy range have involved only the gravitational potential of local sources and utilized temporal variability on the order of 0.1 seconds. Here WEP violation limits are derived from sources with greater distance, faster variability, and larger intervening mass. Specifically, GRB sources with redshifts as high as 6.5 are considered, with variability as fast 0.2 milliseconds, and passing the gravitational potentials of inferred clusters of

\footnotetext{
${ }^{1}$ Work on this chapter has been submitted to Physical Review Letters. Citation: M. Tangmatitham, R. Nemiroff (2019).
} 
galaxies distributed randomly around the line of sight. WEP violation limits are derived from data from GRB 910711, GRB 920229, GRB 021206, GRB 051221, GRB 090429, and GRB 090510. The strongest constraint in the very early universe comes from GRB 090429 which limits $\gamma(500 \mathrm{keV})-\gamma(250 \mathrm{keV})<$ $1.2 \times 10^{-13}$. The strongest overall constraint comes from GRB 090510 which yields a WEP violation limit of $\gamma(30 \mathrm{GeV})-\gamma(1 \mathrm{GeV})<6.6 \times 10^{-16}$. This strongest constraint is not only a new record for WEP violation limit for gammaray photons and in the early universe, but the strongest upper bound for $\Delta \gamma$ that has ever been recorded between any two energy bands.

\subsection{Introduction}

The gravitational equivalence principle has been discussed for over 300 years. Newton's statement of this principle was that mass and weight are locally measured to have an identical ratio for all bodies [30]. Einstein once stated the equivalence principle as "the acceleration imparted to a body by a gravitational field is independent of the nature of the body" [31]. A special case of this, the Weak Equivalence Principle (WEP), applies only to freely falling objects that are not themselves gravitationally bound. In other words, any two free-falling test particles must follow the same trajectory.

In this work, the WEP is explored with photons from gamma-ray bursts (GRBs) of very high energy, rapid variability, and cosmological distances. On their way to the Solar System, each GRB photon must travel through localized gravitational fields of galaxies and clusters of galaxies. Each field induces a temporal lag known as the Shapiro time delay [32]. Parameterized post-Newtonian (PPN) deviations from general relativity (GR) can be described by a factor $\gamma$ where $\gamma=1$ correspond to standard GR [33]. The Shapiro time delay for a 
photon can be expressed as

$$
t_{\text {Shapiro }}=-\frac{1+\gamma}{c^{3}} \int_{D_{S}}^{D_{O}} U(\mathbf{r}(t), t) d r
$$

where the gravitational potential $U$ is integrated along radial coordinate $r$ from a source at light-travel distance $D_{S}$ to the observer at light-travel distance $D_{O}$. For each intermediate mass near the light path of mass $M$, assuming a Keplerian potential this time delay in the frame of the intermediate mass can be approximated as

$$
t_{\text {Shapiro }} \approx(1+\gamma) \frac{G M}{c^{3}} \ln \frac{4 D_{M} D_{M S}}{b^{2}}
$$

where $b$ is the comoving impact parameter of the light with respect to the center of the intervening mass near the light path, $D_{M}$ is the light-travel distance to the point of closest approach to the intervening mass, $D_{M S}$ is the light-travel distance between $D_{M}$ and $D_{S}$.

WEP violations can be parameterized by photons of different energies having nonzero $\Delta \gamma$, resulting from a nonzero $\Delta t_{\text {Shapiro. }}$ A small change in $\Delta \gamma$ in the limit where deviation from GR is small $(\gamma \approx 1)$, can be characterize as

$$
\Delta t_{\text {Shapiro }}=\frac{\Delta \gamma t_{\text {Shapiro }}}{2}
$$

When photons released from a source arrive at a detector at different times, the time difference observed, $\Delta t_{O b s}$, is a combination of the intrinsic time of release from the source $\Delta t_{\text {Release }}$ and the difference in Shapiro time delays experienced by the two photons $\Delta t_{\text {Shapiro. }}$. When observed today, $\Delta t_{O b s}=(1+$ $\left.z_{S}\right) \Delta t_{\text {Release }}+\left(1+z_{M}\right) \Delta t_{\text {Shapiro }}$, where each of the time differences is further expanded by the scale factor $1 / a=1+z$ expressed in terms of redshifts of the source $z_{S}$ and the intermediate mass $z_{M}$.

Even though there is no way to directly measure the $\Delta t_{\text {Release }}$ from a source, we can attribute the upper limit of WEP violations to $\Delta t_{\text {Shapiro }}\left(1+z_{M}\right) \lesssim$ 
$\Delta t_{\text {Obs }}$. Although a nonzero $\left(1+z_{M}\right) \Delta t_{\text {Shapiro }}$ could theoretically cancel out with $\left(1+z_{S}\right) \Delta t_{\text {Release }}$, it would be practically impossible to have it cancel out for every pair of photons having different $\Delta \gamma$. Therefore, by observing $\Delta t_{\text {Obs }}$ we can put an upper limit to $\Delta \gamma$ of the corresponding energies such that $\Delta \gamma \lesssim$ $2 \Delta t_{\text {Obs }} /\left[\left(1+z_{M}\right) t_{\text {Shapiro }}\right]$.

WEP violation limits have been set previously using this method on non-GRB variables. A earlier notable limit on $\Delta \gamma$ in a cosmological setting is from a fast radio burst (FRB) where $\gamma(1.23 \mathrm{GHz})-\gamma(1.45 \mathrm{GHz})<4.36 \times 10^{-9}$ [34]. Similarly, a limit on $\Delta \gamma$ between gravitational wave $(\mathrm{GW})$ and associated electromagnetic signal could put the limit in the order of $\Delta \gamma<10^{-10}$ [35] and, with time delay of less than $1.7 \mathrm{~s}$ in GW 170817, has been measured to be $\gamma_{G W}-\gamma_{E M}<9.8 \times 10^{-8}$ [36].

WEP violation limits involving only GRBs have also been published. Precedents include an analysis of GRB 090510 by Gao et al. [37, resulting in $\gamma_{\mathrm{GeV}}-\gamma_{\mathrm{MeV}}<2 \times 10^{-8}$. Nusser [38] further computed a WEP violation limit from GRB 090510 to be $\gamma_{\mathrm{GeV}}-\gamma_{\mathrm{MeV}}<2.3 \times 10^{-12}$. Both of these used a $\Delta t_{O b s}$ on the order of 0.1 seconds.

All of these previous limits on WEP violations, however, have only taken into account the gravitational potential of our Milky Way galaxy. One practical reason is that the gravitational potential of the Milky Way is known, whereas other potentials along each photon path are not known. In this work, gravitational sources that must exist, statistically, near a random light path to a cosmologically distant object are generated. From a collection of these random light paths, a distribution of expected Shapiro time delays are generated. It will be demonstrated that even the shortest Shapiro time delays expected are, statistically, much larger than the Shapiro time delays created by the Milky Way galaxy or any other local potential. 


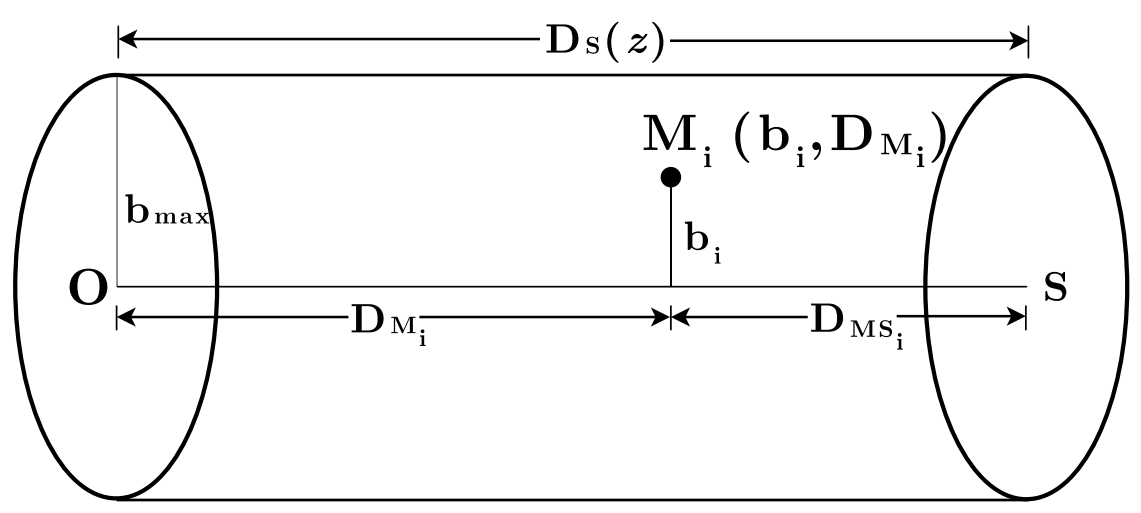

Figure 3.1: For a source located at $D_{S}(z)$, each intermediate mass $\left(M_{i}\right)$ near the light path between the observer $(O)$ and the source $(S)$ is placed randomly at comoving cylindrical coordinate $(r, z)=\left(b_{i}, D_{M i}\right)$.

\subsection{Method}

In this work, a distribution of Shapiro time delays will be simulated from a uniformly random distribution of clusters of galaxies near the light path to a source at redshift $z_{S}$. A standard concordance cosmology is used $\left(\Omega=1, H_{0}=\right.$ $\left.67.74 \mathrm{~km} \mathrm{sec}^{-1} \mathrm{Mpc}^{-1}, \Omega_{\Lambda}=0.6911, \Omega_{m}=0.3089\right)[39]$. For a source located at comoving distance $D_{S}(z)$, the light path to the observer will pass near a series of galaxy clusters, each of mass $M_{i}$, at comoving distance $D_{M_{i}}$ and comoving impact parameters $b_{i}$ (Fig. 3.1).

For $n$ intermediate clusters, the total Shapiro time delay can be expressed as

$$
t_{\text {Shapiro }}=(1+\gamma) \sum_{i=1}^{n}\left(1+z_{M i}\right) \frac{G M_{i}}{c^{3}} \ln \frac{4 D_{M i} D_{M S i}}{b_{i}{ }^{2}}
$$

Each mass $M_{i}$ is placed at comoving cylindrical coordinate $(r, z)=\left(b_{i}, D_{M_{i}}\right)$ with $b_{i} \in\left[0, b_{\max }\right]$ and $D_{M_{i}} \in\left[0, D_{S}(z)\right]$. The cluster distribution within the comoving cylinder is assumed to be randomly distributed with uniform density $\rho_{\text {crit }} \Omega_{C}$. A lower limit of $\Omega_{C}=0.15$, taken from Bahcall et al. [40], is the fraction of mass attributed to clusters of galaxies. 
Each cluster is assumed to have mass between $10^{12}-10^{15} M_{\odot}$ and the frequency distribution described by the mass function of clusters of galaxies [41]

$$
n(>M)=4 \times 10^{-5}\left(\frac{M}{M^{*}}\right)^{-1} e^{-M / M^{*}} \mathrm{~h}^{3} \mathrm{Mpc}^{-3},
$$

where $M^{*}=1.8 \times 10^{14} h^{-1} M_{\odot}$.

The maximum impact parameter, $b_{\max }$, and the comoving distance, $D_{S}(z)$, determine the volume and the amount of mass contained within the cylinder. As $b_{\max }$ increases, clusters that are farther from light path are included, adding smaller contributions per cluster but collectively larger contributions to the Shapiro time delay. A characteristic $b_{\max }=10 \mathrm{Mpc}$ is chosen based on the impact parameters of known gravitational lensing effects from galaxies and clusters on background galaxies [42, 43]. Clusters located within this cutoff radius are known to exhibit weak lensing distortions and therefore must also contribute to the Shapiro time delay.

\subsubsection{Statistical Analysis and Confidence Interval}

Although it is impossible to know the exact gravitational field that an observed pair of photons have passed through, a distribution of expected time delays for photons traveling from redshift $z_{S}$ can be bounded from a simulation to high accuracy. In each simulation, a collection of random light path from a source at redshift $z_{S}$ is generated by filling the comoving cylinder of radius $b_{\max }$ up to critical density. If $t_{5}$ represents the 5 th percentile of Shapiro time delay generated by the simulation, then for over $95 \%$ of random light paths, $t_{5}<t_{\text {Shapiro }}$. Based on the value of $t_{5}$, we can calculate the upper limit for $\Delta \gamma<2 \frac{\Delta t_{\text {Obs }}}{t_{5}}$ with $95 \%$ confidence interval, where $\Delta t_{O b s}$ is the measured minimum variability time scale between photons of different energies for specific GRBs. 


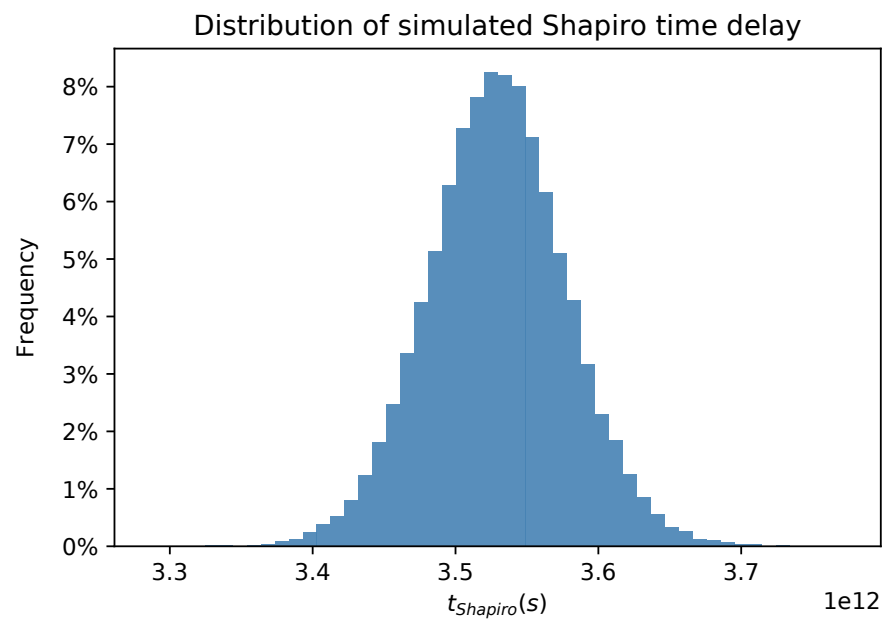

Figure 3.2: Distribution of Shapiro time delay for a source located at $z_{S}=1$ and all clusters within comoving radius of $b_{\max }=10 \mathrm{Mpc}$ are considered.

\subsection{Effects of each parameters on limiting WEP violations}

Based on this method, the Shapiro time delay is obtained from a collection of simulated light paths. From the Shapiro time delay, the limit on $\Delta \gamma$ is then calculated from Eq. 3.1.3. First we explore the effects at which each parameter affect $t_{\text {Shapiro }}$ and conversely the upper bound for $\Delta \gamma$. For each set of parameters, a collection of random light path and intermediate masses is generated into a histogram.

Fig. 3.2 shows the histogram generated from adding the Shapiro time delays from uniformly distributed clusters with the mass distribution given by Eq. 3.2.5 up to redshift $z_{S}=1$, and $b_{\max }=10 \mathrm{Mpc}$. Filling this cylindrical volume up to $\rho_{\text {crit }} \Omega_{C}$ is equivalent to adding $4.10 \times 10^{16} M_{\odot}$ to the volume. The resulting Shapiro time delay has average of $3.55 \times 10^{12} \mathrm{~s}$. Among the distribution of Shaprio time delays generated, $95 \%$ has $t_{\text {Shapiro }}>3.47 \times 10^{12} \mathrm{~s}$. If a detection 
threshold puts a limit $\Delta t_{O b s}<1 \mathrm{~ms}$ from an observation, this corresponds to $\Delta \gamma<5.8 \times 10^{-16}$ with $95 \%$ confidence interval.

\subsubsection{Comparison with WEP effect on local source}

The largest local gravitational source is the Milky Way Galaxy. The presence of local source adds the same amount of Shapiro time delay regardless of the intermediate mass along the line of sight (In higher-order effect, the amount of time delay from local source would depend on the galactic coordinate that a source is coming from). Considering the Milky Way galaxy, the Shapiro time delay can be calculates as follows:

$$
t_{\text {Shapiro, } M W}=2 \frac{G M_{M W}}{c^{3}} \ln \frac{d}{b},
$$

where $M_{M W}=6 \times 10^{11} M_{\odot}, d$ is the light-travel distance to the source $(d=2400 \mathrm{Mpc}$ for source located at $z=1)$, and $b=5 \mathrm{kpc}$. This would give $\Delta t_{M W} / \Delta \gamma=7.8 \times 10^{7} \mathrm{~s}$. Compare this to the histogram of $\Delta t_{\text {Shapiro }}$ contribution from intermediate masses simulated for source at $z=1$ (Figure 3.2) and it is evident that the Shapiro delay contribution from sources along the line of sight is at least 5 order of magnitudes stronger than that from the Milky Way galaxy.

This means that given enough distance to the signal, most of the contribution of the Shapiro time delay comes from the gravitational effects of the intermediate mass and this contribution cannot be ignored.

\subsubsection{Redshift of the Signal}

The comoving volume is defined by a comoving cylinder with radiuds $b_{\max }$ and height defined by the redshift to the source $z_{S}$. As the redshift increases, the light 


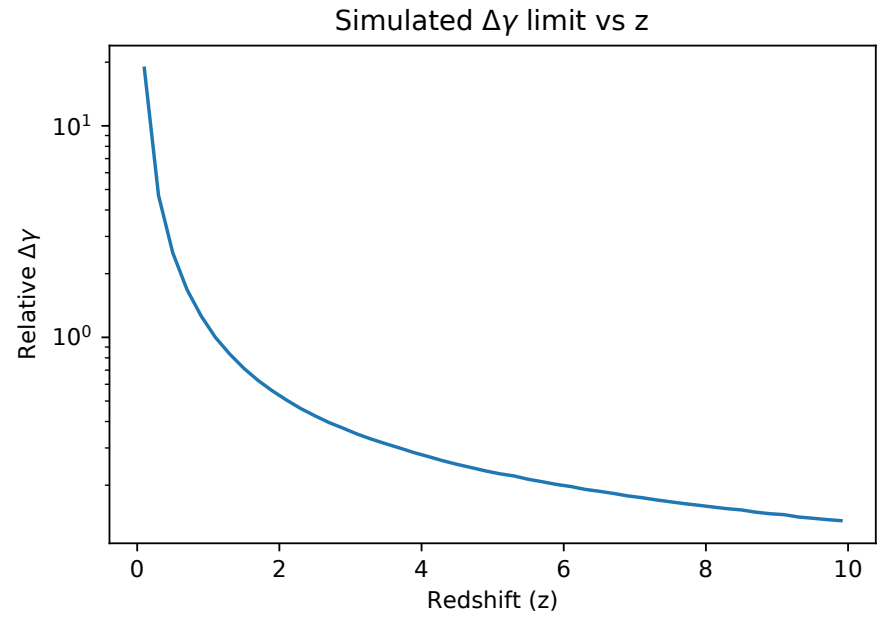

Figure 3.3: Relative $\Delta \gamma$ as a function of redshift $z$ of the signal, normalized to $\Delta \gamma(z=1)$

path must travel through greater light-travel distance to reach the observer. At critical density, this means that the intermediate mass in this cylinder increases as $z_{S}$ increases.

Figure 3.3 shows the plot of predicted $\Delta \gamma$ as the redshift of the source $z$ increases. Benchmark at $z=1$ is used to normalized the relative $\Delta \gamma$ and the maximum comoving radius $b_{\max }=10 \mathrm{Mpc}$ is used in the simulation. From the plot, it is evident that as redshift $z$ increases, the constraints on $\Delta \gamma$ improves due to more mass along the line of sight being added. However, going from $z=1$ to $z=10$ only improves $\Delta \gamma$ by no more than an order of magnitude.

\subsubsection{Cutoff radius}

As cutoff radius $b_{\max }$ of the comoving cylinder increases, the amount of mass and number of clusters required to fill the comoving volume increases. Increasing $b_{\max }$ therefore puts tighter constraints on the $\Delta \gamma$. However, as clusters are placed further away from the line of sight, the contribution of Shapiro time 

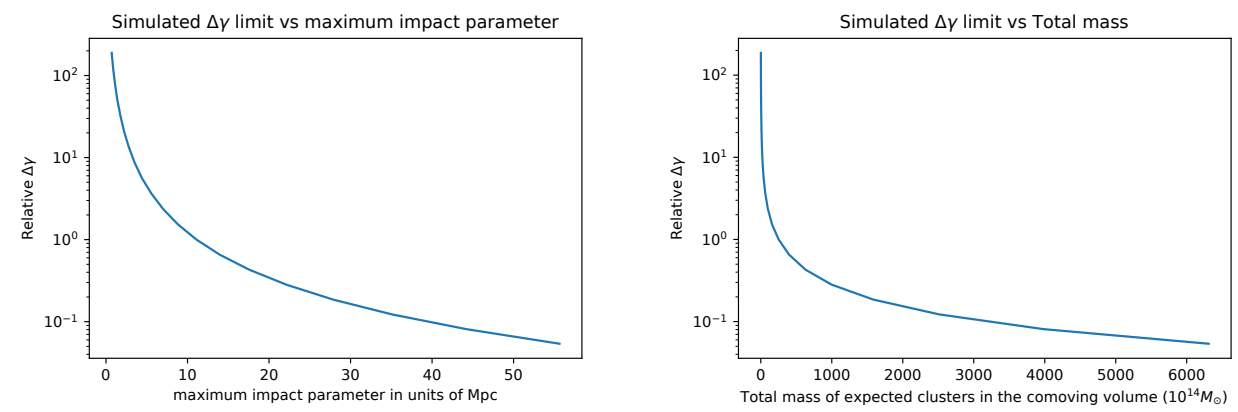

Figure 3.4: Relative $\Delta \gamma$ dependence on the maximum impact parameter in comoving coordinate (left), and the same plot expressed in the mass contained within the comoving volume (right), both normalized to $\Delta \gamma\left(b_{\max }=10 \mathrm{Mpc}\right)$

delay from the intermediate mass decreases. Therefore, as $b_{\max }$ becomes larger, the improvements on $\Delta \gamma$ becomes less and less significant.

Figure 3.4 shows the same plot of relative $\Delta \gamma$ as a function of cutoff radius and as the equivalent mass of clusters included within the cylinder. From the plot, $\Delta \gamma$ improves rapidly as cutoff radius increases, but eventually as $b_{\max }$ gets sufficiently large increasing it only impact the $\Delta \gamma$ ever so slightly.

However, as $b_{\max }$ becomes large, the number of clusters in the cylinder becomes too large and the approximation in Eq. (3.1.2) begins to break down. As $b_{\max }$ approaches infinity it can be considered that the combined gravitational potential of far away masses only contributes to the baseline and constant gravitational potential along the light path. Therefore galaxy clusters outside of $b_{\max }$ can be treated as only contributing to the constant "zero" gravitational potential and only clusters within the cutoff radius $b_{\max }$ contribute towards the gravitational potential fluctuations that resulted in the positive Shapiro time delay.

In this study, $b_{\max }=10 \mathrm{Mpc}$ is used as a benchmark. This value is chosen because it is a characteristic impact parameter often observed in weak lensing. 
If gravitational effects in terms of weak lensing of cluster of galaxies with impact parameters less than $10 \mathrm{Mpc}$ can be easily observed, then it stands to reason that significant Shapiro time delay must also be present for any cluster within $10 \mathrm{Mpc}$ of the light path. For source at $z=1$, using $b_{\max }$ results in the comoving volume of the cylinder of $1.06 \times 10^{6} \mathrm{Mpc}^{3}$. Filling this volume up to $\Omega_{C} \rho_{\text {crit }}$ is equivalent to adding $2.04 \times 10^{16} M_{\odot}$ to the comoving cylinder.

From fig 3.4, it can be seen that reducing $b_{\max }$ from $10 \mathrm{Mpc}$ to $1 \mathrm{Mpc}$ increase $\Delta \gamma$ by 2 order of magnitudes while increasing $b_{\max }$ to $50 \mathrm{Mpc}$ decrease $\Delta \gamma$ by an order of magnitude.

\subsubsection{Cluster Mass Function}

If instead of using cluster mass distribution function (Eq. 3.2.5), an average mass of $M_{\text {avg }}=10^{14} M_{\odot}$ is used, the value of $\Delta \gamma$ constrained only change slightly. Using mass function of clusters at benchmark $z=1, b_{\max }=10 \mathrm{Mpc}$, and

detection threshold $\Delta t_{O b s}=1 \mathrm{~ms}$ yield the value of $\Delta \gamma<5.79 \times 10^{-16}$. Using average mass of $M_{\text {avg }}=10^{14} M_{\odot}$ with the same benchmark parameters yield the value of $\Delta \gamma<5.82 \times 10^{=16}$. Given the nature of upper limit these values are basically the same.

From this result it is evident that changing the mass function impact the Shapiro time delay very slightly. This is partly due to the principle of superposition where multiple small mass or one big mass located at the same location provide identical Shapiro time delay. From this result we can be confident that using wrong model for mass function does not impact the final value of $\Delta \gamma$ much, and that the mass function is a poor constraints on the Shapiro time delay whereas only the total mass contained in the comoving cylinder being considered is a much bigger factor. 


\subsection{Results}

From the Preliminary Analysis, it has been shown that only the $b_{\max }$ and $z$ impacts the final constraints on $\Delta \gamma$. In section 3.3.4 it has been shown that the choice of cluster mass function is mostly irrelevant. Section 3.3.3 shows that the cutoff radius of $b_{\max }=10 \mathrm{Mpc}$ can be used to provide a result that is less than 2 order of magnitudes among reasonable ranges of impact parameters. Lastly, it has been shown in section 3.3.2 that being slightly off in redshift estimation still provides limit on $\Delta \gamma$ that is still way within an order of magnitude.

While this method could limit WEP violation signals between any two particles released simultaneously from the same source, in this work the method is applied to GRBs expected to put the smallest upper bound on WEP violations. These will typically occur for GRBs with the most rapid intrinsic variabilities and the greatest distances. A list of chosen notable GRBs, their reported data, and corresponding limits on WEP-violation limits are shown in Table 3.1. Each GRB has its own details and caveats as described below.

GRB 910711 was measured by BATSE [44] and reported to have a total duration of $16 \mathrm{~ms}$ that persists between energy channel 2 and 4, with energy ranges from $100 \mathrm{KeV}$ to $300 \mathrm{KeV}$ [45]. Unfortunately this GRB did not have a measured redshift and so does not give direct evidence of coherence to the WEP. However, GRB 910711 is a triggered short GRB and so likely occurred at a redshift of $z>0.1$, as have all triggered short GRBs to date [46, 47].

GRB 920229 was detected by BATSE and reported to have a total duration of $190 \mathrm{~ms}$ but an internal flare with a rise time of $0.22 \pm 0.03 \mathrm{~ms}$ [48, 49]. Inspection of Fig 2. of [48] indicates this rise was seen in three energy channels simultaneously with energies 25 - $50 \mathrm{keV}, 50-100 \mathrm{keV}$, and $100-300 \mathrm{keV}$ respectively. Although photons likely came in across this entire energy range, the 
energy range for this rise time as quoted by [49] was between 0.03 and $0.20 \mathrm{MeV}$. GRB 920229, a triggered short GRB, did not have a measured redshift, but as a lower limit, it can be assign a redshift below that of any triggered short GRB to date: $z>0.1$.

GRB 021206 was detected by RHESSI and reported to have a rapid flare of gamma-rays with a duration of about $15 \mathrm{~ms}$ between $2 \mathrm{MeV}$ and "above 10 MeV" [50]. Inspection of Fig. 2 in [50] indicates a conservative energy range estimate of between 3 and $10 \mathrm{MeV}$. Additionally, GRB 021206 had a computed pseudo-redshift of 0.3 , listed as accurate to within a factor of two [50].

GRB 051221A was detected by gamma-ray detectors aboard both KonusWind and Swift. Analysis of the first three peaks in Konus-Wind data indicate that they occur within $4 \mathrm{~ms}$ of each other, in all three energy bands analyzed [51]. Conservatively, the smallest energy gap would be between 0.07 and 0.3 MeV. This GRB had a spectroscopic redshift measured of 0.547 [52].

GRB 090429B is chosen not for its rapid variability but for its high estimated (mean) redshift of 9.4. GRB 090429B was detected by the gamma-ray detector on Swift and contained three temporal peaks with a combined measured duration of $5 \mathrm{sec}$ [53]. Assuming a Small Magellanic Cloud dust law yields a photometric redshift bounds (90\% confidence level) of $9.06<z<9.52$ [54]. However, the lowest photometric redshift estimated from a high-z dust law gives $z>6.5$ at $99 \%$ confidence. To be conservative, this lowest redshift estimate is used here. The measured $t_{90}$ duration of the burst was $5.2 \mathrm{sec}$ [54], however, a temporal lag in the cross-correlation between an energy channel of 15 - $25 \mathrm{keV}$ and an energy channel of 50 - $100 \mathrm{keV}$ was found to be $1200 \mathrm{~ms}$ (95\% confidence level) [54].

GRB 090510 was detected by the Fermi satellite including its Large Area Telescope. Subsequent analysis resulted in a reported variability of $1.55 \mathrm{~ms}$ over an energy range from $1.58 \mathrm{GeV}$ to about $24.7 \mathrm{GeV}$ [55]. The $2-\sigma$ lower limit on 


\begin{tabular}{|l|c|c|c|c|c|c|c|}
\hline Name & Instrument & $\begin{array}{c}\Delta t_{\text {Obs }} \\
(\mathrm{ms})\end{array}$ & $\begin{array}{c}E_{\text {min }} \\
(\mathrm{MeV})\end{array}$ & $\begin{array}{c}E_{\text {max }} \\
(\mathrm{MeV})\end{array}$ & $z$ & $\begin{array}{c}\Delta \gamma \\
\left(E_{\text {max }}, E_{\text {min }}\right)\end{array}$ & $\begin{array}{c}\Delta \gamma_{r m s}^{\dagger} \\
\left(E_{\text {max }}, E_{\text {min }}\right)\end{array}$ \\
\hline GRB 910711 & BATSE & 16 & 0.1 & 0.3 & $>0.1$ (assumed) & $1.6 \times 10^{-13}$ & $8.3 \times 10^{-11}$ \\
GRB 920229 & BATSE & 0.22 & 0.03 & 0.2 & $>0.1$ (assumed) & $2.1 \times 10^{-15}$ & $1.1 \times 10^{-12}$ \\
GRB 021206 & RHESSI & 4.8 & 3 & 10 & $>0.15$ (pseudo) & $2.8 \times 10^{-14}$ & $2.0 \times 10^{-11}$ \\
GRB 051221A & Konus-Wind & 4 & 0.07 & 0.3 & 0.547 (spectral) & $4.7 \times 10^{-15}$ & $5.9 \times 10^{-12}$ \\
GRB 090429 & Swift & 1200 & 0.25 & 0.5 & 6.5 (pseudo) & $1.2 \times 10^{-13}$ & $1.1 \times 10^{-10}$ \\
GRB 090510 & Fermi & 1.0 & 1580 & 24,700 & 0.897 (spectral) & $6.6 \times 10^{-16}$ & $7.6 \times 10^{-13}$ \\
\hline
\end{tabular}

$\dagger$ Obtained from distribution of root mean squared $t_{\text {Shapiro }}$ difference compared to the average

Table 3.1: A Table of data and WEP-violation limits for rapidly fluctuating and distant GRBs.

this GRB's spectroscopic redshift is 0.897 [56].

These WEP-violation limits assume that the mass distribution of clusters of galaxies given by $(3.2 .5)$ have been relatively unchanged from $z=6.5$ to today. This simulation also assumed that the Universe is homogeneous in the comoving coordinate across the volume within the cutoff radius of $b_{\max }=10 \mathrm{Mpc}$.

These are the strongest limits yet found on WEP violations on high energy photons, and at cosmological distances. Further, the limit at $z>6.5$ is the only limit when the universe was only a fraction of its present age. The strongest overall limit comes from GRB 090510 which is the strongest limit on WEP violation between any energy scale and anywhere in the universe.

If WEP-violation limit is expanded onto two photons of the same energy, then the current limit has been measured based photons of two different circular polarizations [57] [58] which reach an upper bound of $\Delta \gamma_{p}<1.06 \times 10^{-28}$ [58]. Using the redshift $z=0.382$ and the time limit required to maintain linear polarization of $\Delta t_{\text {Obs }}=6 \times 10^{-20} s$, the best upper limit between any pair of photons is $\Delta \gamma_{p}<1.09 \times 10^{-31}$. 
Work done by Nusser attempted to explore the large-scale ( $\gtrsim 10 \mathrm{Mpc})$ structure (LSS) fluctuations [38]. Nusser argued that since the gravitational potential $U^{L S}$ fluctuates around zero, the cosmological Shapiro shift may acquire negative as well as positive values. It is possible to account for this by setting the average amount of Shapiro time delay in a given distribution to be zero. Then the amount of time delay can be either positive or negative, suggesting that it might be possible for the higher energy photons to be faster in some case and slower in another compared to photons of lower energy.

If this zero Shapiro delay baseline were to be applied to the benchmark $(z=1)$, then it is equivalent to setting the mean value of the histogram in Fig. 3.2 to zero. Finding the 5th percentile of the root mean squared time different from this new histogram suggests that, for $95 \%$ of the time, the Shapiro time delay would differ from the average value by at least $3.6 \times 10^{9} \mathrm{~s}$. For $\Delta t_{O b s}=1$ ms, his instead constraints WEP violation to $\Delta \gamma_{r m s}<5.5 \times 10^{-13}$, as opposed to the $\Delta \gamma<5.8 \times 10^{-16}$ as shown in Section 3.3. Last column of Table 3.1 shows the value of $\Delta \gamma_{r m s}$ by considering the 5 th percentile of the root mean squared time delay compared to the average value. This results in a more conservative limit on the WEP violations that are around 3 order of magnitudes larger.

In summary, by considering random galaxy clusters near the light path to the expected density, a distribution of gravitational potentials, and hence Shapiro time delays, along a random light path was simulated. We have shown that the contribution to the Shapiro time delay from these mass to any random light path is magnitudes greater than any local gravitational potential could provide, leading to an upper bound of $\Delta \gamma$ of WEP violations that is decreased significantly. This method can also be applied to future observations with potentially even finer time variability or across larger cosmological distances. Most importantly, this method is not unique to GRBs, but can be applied to any event 
that two particles are known to have been emitted, near simultaneously, across cosmological distances. 


\section{Chapter 4}

\section{Conclusion}

\section{Location, orbit and energy of a meteoroid im- pacting the moon during the Lunar Eclipse of January 21, 2019}

By combining images taken by amateur astronomer across the globe, we are able to extract location, parallax, and photometric data that could help identify the impact site of meteor impact event that occurred during the lunar eclipse of January 21, 2019. The sets of circumstances that occurred during total lunar eclipse allow one of the best chance possible to perform lunar parallax measurements with precision in the order of $200 \mathrm{~km}$. Using the combined images from multiple locations we are able to construct a geometrical method to find the selenographic coordinate of the flash at higher precision allowed by visual method.

Future discovery of the crater left by the impact, if resolvable, and its comparison with the predictions of the models involved in this work will greatly contribute to the improvement and testing the validity of the Gravitational Ray Tracing technique. 
The success of the GRT technique will not only confirm the result provided by this work, but can also serve as a foundation to which future work in similar area can progress. In the future, technique similar to GRT could be applied to observations on other solar system objects such as impacts on Jupiter or its moon.

The special set of circumstances that lead to this event have been quite rare. However, it is conceivable that with increasing interest in astronomy and availability of digital cameras another impact on the moon during the future lunar eclipses might again be observed simultaneously among many observers. This research should be able to set as a framework to the level of analysis that can be obtained from future observations made by amateur and professional astronomers alike.

\section{Testing the Weak Equivalence Principle with Cos- mological Gamma Ray Bursts}

By considering random galaxy clusters near the light path to the expected density, a distribution of gravitational potentials and hence the Shapiro time delay along a random light path was simulated. We have shown that the contribution to the Shapiro time delay from these mass expected from a random light path is magnitudes greater than any local gravitational potential could provide, leading to an upper bound of $\Delta \gamma$ of WEP violations that has never before been reached.

The effects of each parameters on limiting the WEP violations has been explored. This will shed light to the kind of future observations that will provide even better constraints. For example, GRB with higher redshift and finer time variability between much wider energy bands will constrain the limit to the violations even further. 
This method is not unique only to GRBs, but can be applied to any event that two particles are known to have been emitted near simultaneously across cosmological distances. For example, if the observed time difference between future Gravitational waves and its optical counterparts have been measured, this technique can be applied to reach a better constraints on the WEP violations that would otherwise be possible based purely on just local gravitational field.

If a real violation to the WEP should ever one day be confirmed for cosmological sources, then the author believes that the result shown here should already prove why the method described in this work should be the superior method to estimate the level of WEP violations permissible by General Relativity. 


\section{References}

[1] R. J. Nemiroff and J. T. Bonnell, "Astronomy Picture of the Day: http://antwrp.gsfc.nasa.gov/apod/astropix.html," in American Astronomical Society Meeting Abstracts, vol. 27 of Bulletin of the American Astronomical Society, p. 1291, Dec. 1995.

[2] J. Overduin, F. Everitt, J. Mester, and P. Worden, "The Science Case for STEP," Advances in Space Research, vol. 43, pp. 1532-1537, May 2009.

[3] J. M. Madiedo, J. M. Trigo-Rodríguez, J. L. Ortiz, and N. Morales, "Robotic Systems for Meteor Observing and Moon Impact Flashes Detection in Spain," Advances in Astronomy, vol. 2010, p. 167494, 2010.

[4] C. Sigismondi and G. Imponente, "The Observation of Lunar Impacts," WGN, Journal of the International Meteor Organization, vol. 28, pp. 5457, June 2000.

[5] C. Sigismondi and G. Imponente, "The Observation of Lunar Impacts. Part II," WGN, Journal of the International Meteor Organization, vol. 28, pp. 230-232, Dec. 2000.

[6] G. Neukum, B. A. Ivanov, and W. K. Hartmann, "Cratering Records in the Inner Solar System in Relation to the Lunar Reference System," Space Sci. Rev., vol. 96, pp. 55-86, Apr. 2001. 
[7] B. A. Ivanov, "Mars/Moon Cratering Rate Ratio Estimates," Space Sci. Rev., vol. 96, pp. 87-104, Apr. 2001.

[8] B. A. Ivanov, "Earth/Moon impact rate comparison: Searching constraints for lunar secondary/primary cratering proportion," Icarus, vol. 183, pp. 504-507, Aug. 2006.

[9] J. Gallant, B. Gladman, and M. Cuk, "Current bombardment of the Earth-Moon system: Emphasis on cratering asymmetries," Icarus, vol. 202, pp. 371-382, Aug. 2009.

[10] P. Brown, R. E. Spalding, D. O. ReVelle, E. Tagliaferri, and S. P. Worden, "The flux of small near-Earth objects colliding with the Earth," Nature, vol. 420, pp. 294-296, Nov. 2002.

[11] J. B. Hartung, "Giordano bruno, the june 1975 meteoroid storm, encke, and other taurid complex objects," Icarus, vol. 104, no. 2, pp. 280-290, 1993.

[12] J. Keller, N. Petro, R. Vondrak, et al., "The lunar reconnaissance orbiter mission-six years of science and exploration at the moon," Icarus, vol. 273, pp. 2-24, 2016.

[13] R. Suggs, D. Moser, W. Cooke, and R. Suggs, "The flux of kilogram-sized meteoroids from lunar impact monitoring," Icarus, vol. 238, pp. 23-36, 2014.

[14] J. M. Madiedo, J. L. Ortiz, N. Morales, and J. Cabrera-Caño, "A large lunar impact blast on 2013 September 11," Monthly Notices of the Royal Astronomical Society.

[15] J. L. Ortiz, P. V. Sada, L. R. Bellot Rubio, F. J. Aceituno, J. Aceituno, P. J. Gutiérrez, and U. Thiele, "Optical detection of meteoroidal impacts on the Moon," Nature, vol. 405, pp. 921-923, June 2000. 
[16] J. L. Ortiz, J. A. Quesada, J. Aceituno, F. J. Aceituno, and L. R. Bellot Rubio, "Observation and Interpretation of Leonid Impact Flashes on the Moon in 2001," Astrophys. J., vol. 576, pp. 567-573, Sept. 2002.

[17] J. L. Ortiz, F. J. Aceituno, J. A. Quesada, J. Aceituno, M. Fernández, P. Santos-Sanz, J. M. Trigo-Rodríguez, J. Llorca, F. J. Martín-Torres, P. Montañés-Rodríguez, and E. Pallé, "Detection of sporadic impact flashes on the Moon: Implications for the luminous efficiency of hypervelocity impacts and derived terrestrial impact rates," Icarus, vol. 184, pp. 319-326, Oct. 2006.

[18] J. L. Ortiz, J. M. Madiedo, N. Morales, P. Santos-Sanz, and F. J. Aceituno, "Lunar impact flashes from Geminids: analysis of luminous efficiencies and the flux of large meteoroids on Earth," Monthly Notices of the Royal Astronomical Society.

[19] J. M. Madiedo, J. L. Ortiz, N. Morales, and J. Cabrera-Caño, "MIDAS: Software for the detection and analysis of lunar impact flashes," Planet. Space Sci., vol. 111, pp. 105-115, June 2015.

[20] J. M. Madiedo, J. L. Ortiz, F. Organero, L. Ana-Hernández, F. Fonseca, N. Morales, and J. Cabrera-Caño, "Analysis of Moon impact flashes detected during the 2012 and 2013 Perseids," Astronom. Astrophys., vol. 577, p. A118, May 2015.

[21] M. Yanagisawa and N. Kisaichi, "Lightcurves of 1999 Leonid Impact Flashes on the Moon," Icarus, vol. 159, pp. 31-38, Sept. 2002.

[22] E. M. Xilouris, A. Z. Bonanos, I. Bellas-Velidis, P. Boumis, A. Dapergolas, A. Maroussis, A. Liakos, I. Alikakos, V. Charmandaris, G. Dimou, A. Fytsilis, M. Kelley, D. Koschny, V. Navarro, K. Tsiganis, and K. Tsinganos, 
"NELIOTA: The wide-field, high-cadence, lunar monitoring system at the prime focus of the Kryoneri telescope," Astronom. Astrophys., vol. 619, p. A141, Nov. 2018.

[23] P. Duffett-Smith, Practical astronomy with your calculator. The Pitt Building, Trumpington Street, Cambridge CB2 1RP: The Press Syndicate of the University of Cambridge, 1979.

[24] J. I. Zuluaga and M. Sucerquia, "Towards a theoretical determination of the geographical probability distribution of meteoroid impacts on Earth," Monthly Notices of the Royal Astronomical Society.

[25] L. R. Bellot Rubio, J. L. Ortiz, and P. V. Sada, "Luminous Efficiency in Hypervelocity Impacts from the 1999 Lunar Leonids," Astrophys. J. Lett., vol. 542, pp. L65-L68, Oct. 2000.

[26] J. Deglint, F. Kazemzadeh, D. Cho, D. A. Clausi, and A. Wong, "Numerical demultiplexing of color image sensor measurements via non-linear random forest modeling," Scientific reports, vol. 6, p. 28665, 2016.

[27] D. E. Gault, "Impact cratering," in A Primer in Lunar Geology (R. Greeley and P. H. Schultz, eds.), July 1974.

[28] H. J. Melosh, Impact cratering: A geologic process. 1989.

[29] A. V. Moorhead, R. C. Blaauw, D. E. Moser, M. D. Campbell-Brown, P. G. Brown, and W. J. Cooke, "A two-population sporadic meteoroid bulk density distribution and its implications for environment models," Monthly Notices of the Royal Astronomical Society, vol. 472, no. 4, pp. 3833-3841, 2017.

[30] I. Newton, Philosophiae Naturalis Principia Mathematica. Auctore Js. Newton. 1687. 
[31] A. Einstein, The Meaning of Relativity. 2004.

[32] I. I. Shapiro, "Fourth Test of General Relativity," Physical Review Letters, vol. 13, pp. 789-791, Dec. 1964.

[33] C. M. Will, "The confrontation between general relativity and experiment," Living Reviews in Relativity, vol. 9, p. 3, Mar 2006.

[34] J.-J. Wei, H. Gao, X.-F. Wu, and P. Mészáros, "Testing Einstein's Equivalence Principle With Fast Radio Bursts," Physical Review Letters, vol. 115, p. 261101, Dec. 2015.

[35] X.-F. Wu, H. Gao, J.-J. Wei, P. Mészáros, B. Zhang, Z.-G. Dai, S.-N. Zhang, and Z.-H. Zhu, "Testing Einstein's weak equivalence principle with gravitational waves," Physical Review D, vol. 94, p. 024061, July 2016.

[36] S. Boran, S. Desai, E. O. Kahya, and R. P. Woodard, "GW170817 falsifies dark matter emulators," Physical Review D, vol. 97, p. 041501, Feb. 2018.

[37] H. Gao, X.-F. Wu, and P. Mészáros, "Cosmic Transients Test Einstein's Equivalence Principle out to GeV Energies," Astrophysical Journal, vol. 810, p. 121, Sept. 2015.

[38] A. Nusser, "On Testing the Equivalence Principle with Extragalactic Bursts," Astrophysical Journal Letters, vol. 821, p. L2, Apr. 2016.

[39] Planck Collaboration, P. A. R. Ade, N. Aghanim, M. Arnaud, M. Ashdown, J. Aumont, C. Baccigalupi, A. J. Banday, R. B. Barreiro, J. G. Bartlett, and et al., "Planck 2015 results. XIII. Cosmological parameters," Astronomy and Astrophysics, vol. 594, p. A13, Sept. 2016.

[40] N. A. Bahcall, F. Dong, P. Bode, R. Kim, J. Annis, T. A. McKay, S. Hansen, J. Schroeder, J. Gunn, J. P. Ostriker, M. Postman, R. C. Nichol, C. Miller, 
T. Goto, J. Brinkmann, G. R. Knapp, D. O. Lamb, D. P. Schneider, M. S. Vogeley, and D. G. York, "The Cluster Mass Function from Early Sloan Digital Sky Survey Data: Cosmological Implications," Astrophysical Journal, vol. 585, pp. 182-190, Mar. 2003.

[41] N. A. Bahcall and R. Cen, "The mass function of clusters of galaxies," Astrophysical Journal, Letters, vol. 407, pp. L49-L52, Apr. 1993.

[42] R. Mandelbaum, A. Slosar, T. Baldauf, U. Seljak, C. M. Hirata, R. Nakajima, R. Reyes, and R. E. Smith, "Cosmological parameter constraints from galaxy-galaxy lensing and galaxy clustering with the SDSS DR7," Monthly Notices of the RAS, vol. 432, pp. 1544-1575, June 2013.

[43] A. Leauthaud, S. Saito, S. Hilbert, A. Barreira, S. More, M. White, S. Alam, P. Behroozi, K. Bundy, J. Coupon, T. Erben, C. Heymans, H. Hildebrandt, R. Mandelbaum, L. Miller, B. Moraes, M. E. S. Pereira, S. A. RodríguezTorres, F. Schmidt, H.-Y. Shan, M. Viel, and F. Villaescusa-Navarro, "Lensing is low: cosmology, galaxy formation or new physics?," Monthly Notices of the RAS, vol. 467, pp. 3024-3047, May 2017.

[44] G. J. Fishman, C. A. Meegan, R. B. Wilson, T. A. Parnell, W. S. Paciesas, G. N. Pendleton, H. S. Hudson, J. L. Matteson, L. E. Peterson, T. L. Cline, B. J. Teegarden, and B. E. Schaefer, "The BATSE Experiment for the GRO - Solar Flare Hard X-Ray and Gamma-Ray Capabilities," in Bulletin of the American Astronomical Society, vol. 21 of Bulletin of the AAS, p. 860, Mar. 1989.

[45] P. N. Bhat, G. J. Fishman, C. A. Meegan, R. B. Wilson, M. N. Brock, and W. S. Paciesas, "Evidence of sub-millisecond structure in a gamma-ray burst," Nature, vol. 359, p. 217, Sept. 1992. 
[46] E. Berger, "Short-Duration Gamma-Ray Bursts," Bulletin of the AAS, vol. 52, pp. 43-105, Aug. 2014.

[47] J. Greiner, "Localized GRBs web table," 2019.

[48] B. E. Schaefer and K. C. Walker, "A Gamma-Ray Burst with a 220 Microsecond Rise Time and a Sharp Spectral Cutoff," Astrophysical Journal, Letters, vol. 511, pp. L89-L92, Feb. 1999.

[49] B. E. Schaefer, "Severe Limits on Variations of the Speed of Light with Frequency," Physical Review Letters, vol. 82, pp. 4964-4966, June 1999.

[50] S. E. Boggs, C. B. Wunderer, K. Hurley, and W. Coburn, "Testing Lorentz Invariance with GRB 021206," Astrophysical Journal, Letters, vol. 611, pp. L77-L80, Aug. 2004.

[51] M. Rodríguez Martínez, T. Piran, and Y. Oren, "GRB 051221A and tests of Lorentz symmetry," Journal of Cosmology and Astroparticle Physics, vol. 5, p. 017, May 2006.

[52] A. M. Soderberg, E. Berger, M. Kasliwal, D. A. Frail, P. A. Price, B. P. Schmidt, S. R. Kulkarni, D. B. Fox, S. B. Cenko, A. Gal-Yam, E. Nakar, and K. C. Roth, "The Afterglow, Energetics, and Host Galaxy of the Short-Hard Gamma-Ray Burst 051221a," Astrophysical Journal, vol. 650, pp. 261-271, Oct. 2006.

[53] T. N. Ukwatta, S. D. Barthelmy, P. A. Evans, N. Gehrels, C. B. Markwardt, K. L. Page, D. M. Palmer, B. A. Rowlinson, M. H. Siegel, M. Stamatikos, and L. Vetere, "GRB 090429B: Swift detection of a burst.," GRB Coordinates Network, vol. 9281, 2009. 
[54] A. Cucchiara, A. J. Levan, D. B. Fox, N. R. Tanvir, T. N. Ukwatta, E. Berger, T. Krühler, A. Küpcü Yoldaş, X. F. Wu, K. Toma, J. Greiner, F. E. Olivares, A. Rowlinson, L. Amati, T. Sakamoto, K. Roth, A. Stephens, A. Fritz, J. P. U. Fynbo, J. Hjorth, D. Malesani, P. Jakobsson, K. Wiersema, P. T. O'Brien, A. M. Soderberg, R. J. Foley, A. S. Fruchter, J. Rhoads, R. E. Rutledge, B. P. Schmidt, M. A. Dopita, P. Podsiadlowski, R. Willingale, C. Wolf, S. R. Kulkarni, and P. D'Avanzo, "A Photometric Redshift of z " 9.4 for GRB 090429B," Astrophysical Journal, vol. 736, p. 7, July 2011.

[55] R. J. Nemiroff, R. Connolly, J. Holmes, and A. B. Kostinski, "Bounds on Spectral Dispersion from Fermi-Detected Gamma Ray Bursts," Physical Review Letters, vol. 108, p. 231103, June 2012.

[56] A. A. Abdo, M. Ackermann, M. Ajello, K. Asano, W. B. Atwood, M. Axelsson, L. Baldini, J. Ballet, G. Barbiellini, M. G. Baring, and et al., "A limit on the variation of the speed of light arising from quantum gravity effects," Nature, vol. 462, pp. 331-334, Nov. 2009.

[57] C. Yang, Y.-C. Zou, Y.-Y. Zhang, B. Liao, and W.-H. Lei, "Testing the Einstein's equivalence principle with polarized gamma-ray bursts," Monthly Notices of the RAS, vol. 469, pp. L36-L38, July 2017.

[58] O. Bertolami and R. G. Landim, "Cosmic transients, Einstein's Equivalence Principle and dark matter halos," Physics of the Dark Universe, vol. 21, pp. 16-20, Sept. 2018. 


\section{Appendix A}

\section{Copyright documentations}

\section{A.1 Copyright documentation of Chapter 2}

Location, orbit and energy of a meteoroid impacting the moon during the Lunar Eclipse of January 21, 2019 has been submitted to the journal Icarus. At the time of this dissertation's publication it has not yet been published. Therefore the authors involved in this paper still retain all the copyright to this work.

Below is the copyright documentation from the contributing author of the paper, Jorge Zuluaga, along with the certification and contribution to the improved version of the manuscript on the Lunar Impact of January 21, 2019. 
Medellín, 16 April 2019

To whom it may concern,

With this letter, and as first author of the paper entitled "Location, orbit and energy of a meteoroid impacting the moon during the Lunar Eclipse of January 21, 2019", I want certify the authorship and describe the contributions of Mr. Matipon Tangmatitham to manuscript.

This paper resulted of a timely collaboration between professional and amateur astronomers from Colombia and the Dominican Republic after the observation of an impact on the Moon during the Lunar Eclipse of January 21, 2019.

Being a topic of great interest for the astronomical community, we submitted the paper to the arXiv and to the Icarus journal (where today is still under review) few days after the event. This first version of the manuscript did not include Mr. Tangmatitham as co author.

After knowing about our work, Mr. Tangmatitham contacted us, offering additional data he has collected on the lunar impact of January 21, 2019 from amateurs around the world, but moreover offering new and fresh ideas of how to integrate those additional data to our work.

After a fruitful collaboration during the last two months, we have achieved at improving substantially our original work with the decisive contributions of Mr. Tangmatitham.

As a result we have prepared and submitted a replacement to the arXiv of the paper (that will appear on 17 April at 19:00 UTC-5) and will submit soon the revised version (including Mr. Mr. Tangmatitham as co author) to the journal.

Recepción de correspondencia: calle 70 No. 52 - 21 | Apartado Aéreo 1226 | Dirección: calle 67 No. 53 - 108

Conmutador: [57+4] 2198332 |Correo electrónico: atencionciudadano@audea.edu.co | NIT 890980040-8

Medellin - Colombia|Todos los Derechos Reservados O 2011 


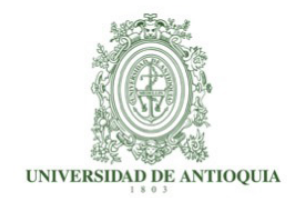

Mr. Tangmatitham contributions to the paper have been diverse and instrumental for the new version of the manuscript.

He not only provided original data and served as a contact person with the amateurs who took the pictures we now include in our paper, but also introduced several key techniques to the paper and developed computational codes to implement them. $\mathrm{He}$ contributed significantly to the content of several new sections and write entirely the appendix on the determination of the distance to the moon using the compiled observations. He also prepare several of the new figures in the manuscript. Moreover, during our discussions in the last months, he have provided insightful criticism to the already included results as well to new ones obtained for our co authors.

His contributions to the new version of the manuscript have been so numerous and important that we, all the former co authors, have decided he should be the second author of the improved version of the manuscript.

Although this situation is rather uncommon in scientific collaborations, it should also be understood that observing and analysing almost in real time an impact on the moon, observed by thousands if not millions of casual observers, is also very uncommon.

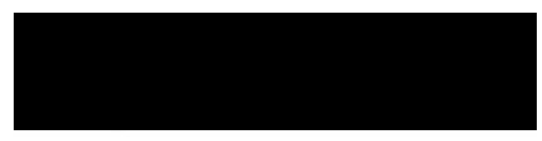

JORGE IVÁN ZULUAGA CALLEJAS

Solar Earth and Planetary Physics Group

Institute of Physics - Faculty of Exact and Natural Sciences - Universidad de Antioquia

Recepción de correspondencia: calle 70 No. 52 - 21 | Apartado Aéreo 1226 | Dirección: calle 67 No. 53 - 108 Conmutador: [57+4] 2198332 | Correo electrónico: atencionciudadano@udea.edu.co| | NIT 890980040-8

Medellin - Colombia |Todos los Derechos Reservados 02011

Below are the screenshot of the emails displaying copyright consent from the 
amateur photographers involved in this project.

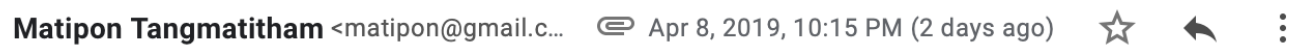
to gregoryhogan, Schraebler, Libor, robert.eder, horalek.peter, drhemmerich, JORGE -

Hi all,

You are receiving this email because you have sent in your image of the lunar impact event during the total lunar eclipse of January 21st.

I would like to give you an update on this project. I have been collaborating with Jorge Ivan Zuluaga from University of Antioquia, Colombia into integrating additional data provided by you to perform further analysis on this unique historical event. With the combined data we are able to obtained improved photometry measurement to find the magnitude of the flash and narrow down the selenographic coordinate on the lunar surface where this happened. Potentially, lunar-orbiting satellite can search this location and perhaps find a new impact crater from this event. These data is further used to estimate the speed, angle, energy, size of the impactor, and potential crater radius.

Also, with the combined data taken from locations across the globe we were able to calculate parallax measurements on the moon with precision as accurate as a few hundred kilometers. This is potentially among the most accurate lunar parallax measurements ever been made, if not the most accurate.

You can read more about the work from the attached pdf file. This work has been submitted to Icarus journal and it is under review. Note that the manuscript cannot yet be shared publicly until the reviewing process is completed. Once the review process is completed, an open-access eprints version will be uploaded to arxiv.org.

This work can never be achieved without your contribution. My collaborator is going to be giving a presentation on this work as an example on the collaboration between professional and amateur astronomers during the 100 years IAU flagship event in Brussels on April 13th.

I would also like to confirm with you all again regarding the consent and permission for your images to be used in this work. As part of keeping up with the scientific tradition of reproducibility, we are also making all our codes and data publicly available. For this reason, a copy of your raw images will be post on a companion GitHub repository under cc-by-nc-sa 4.0 license.

You can access the repository here: https://github.com/seap-udea/MoonFlashes

Please confirm by replying to this email that you are giving your permission for your raw images to be used and a copy to be uploaded to GitHub repository.

Dr. Fritz Helmut Hemmerich

to me -

3:45 AM (11 hours ago) 计 h :

Off course you are allowed to use and publish my raw and processed images from this event, respecting the appropriate image credits.

Cheers Fritz

Greg Hogan

to me -

Apr 9, 2019, 8:25 AM (1 day ago) 究 $h$ :

Permission granted. Thanks so much for the update!

Libor Haspl

to gregoryhogan, Schraebler, robert.eder, horalek.peter, drhemmerich, JORGE, me -

Yes, I confirm. 


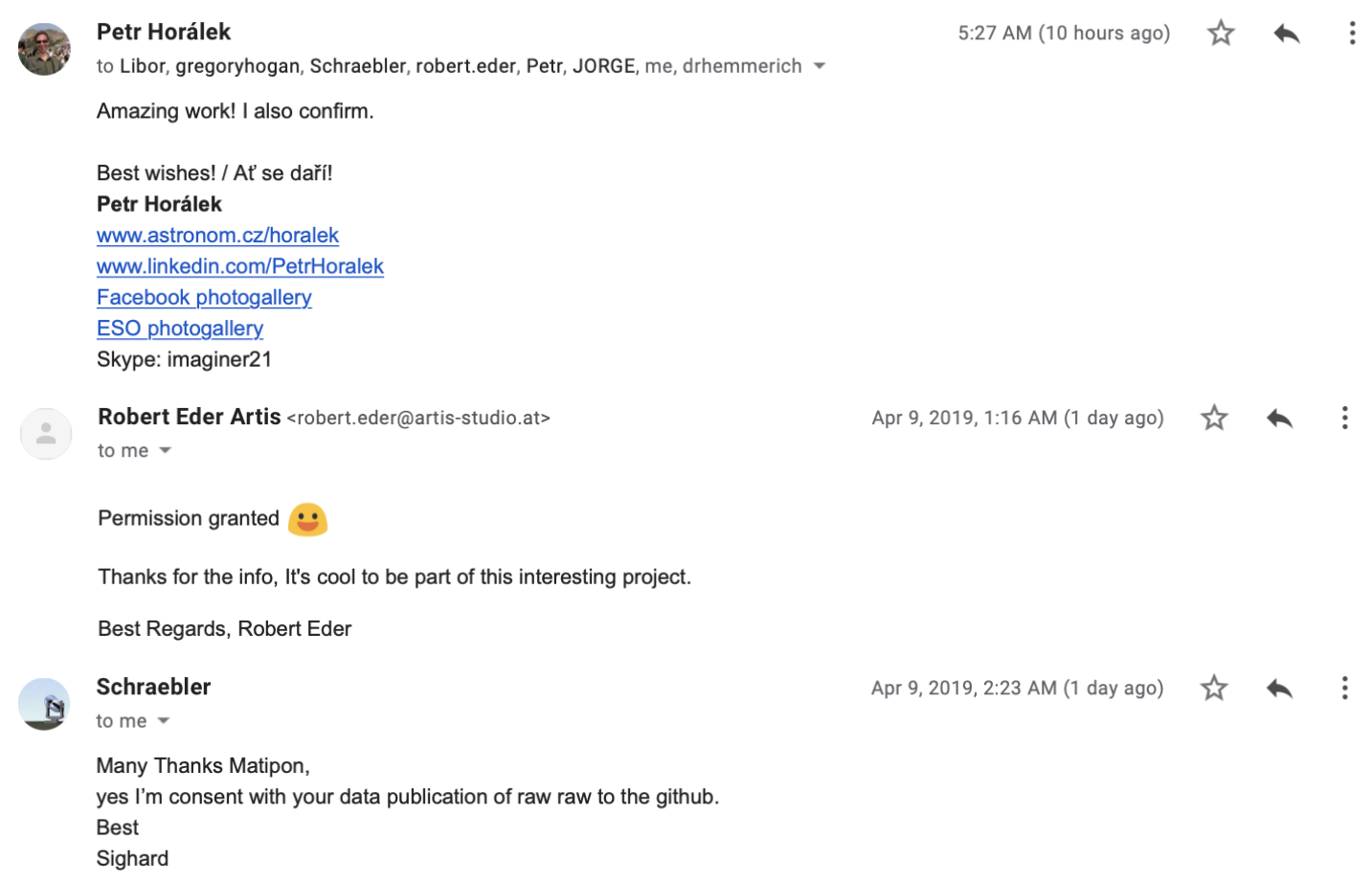

\section{A.2 Copyright documentation of Chapter 3}

Testing the Weak Equivalence Principle with Cosmological Gamma Ray Bursts has been submitted to the journal Physical Review Letters. At the time of this dissertation's publication it has not yet been published. Therefore the authors involved in this paper still retain all the copyright to this work. 